\title{
Stereoselective synthesis of the glycosidase inhibitor australine through a one-pot, double cyclization strategy
}

Celia Ribes, ${ }^{\mathrm{a}}$ Eva Falomir, ${ }^{\mathrm{a}}$ Miguel Carda, ${ }^{\mathrm{a}}$ and J. Alberto Marco ${ }^{\mathrm{b}}$

${ }^{a}$ Departamento de Química Inorgánica y Orgánica, Universidad Jaume I, E-12080 Castellón, Spain; and ${ }^{b}$ Departamento de Química Orgánica, Universidad de Valencia, E46100 Burjassot, Valencia, Spain

For Senior Author:

J.A. Marco:

-Mailing address: as above

-Phone No.: 34-96-3544337

-Fax No.: 34-96-3544328

-E-Mail: alberto.marco@uv.es

\section{Supporting Information}

\section{Contents:}

S-2: General features and reaction scheme

S-3/S-10: General experimental conditions

S-11/S-12: Tables with compared NMR data

S-13/S-32: ${ }^{1} \mathrm{H}$ and ${ }^{13} \mathrm{C}$ NMR spectra of new compounds 
General Features. NMR spectra were recorded at $500 \mathrm{MHz}\left({ }^{1} \mathrm{H} \mathrm{NMR}\right)$ and $125 \mathrm{MHz}\left({ }^{13} \mathrm{C} \mathrm{NMR}\right)$ in $\mathrm{CDCl}_{3}$ solution at $25^{\circ} \mathrm{C}$ with the solvent signals as internal reference (the NMR spectra of australine 1 were measured in $\left.\mathrm{D}_{2} \mathrm{O}\right) .{ }^{13} \mathrm{C}$ NMR signal multiplicities were determined with the APT pulse sequence. Mass spectra were run in the $\mathrm{EI}(70 \mathrm{eV})$ or the $\mathrm{FAB}$ ( $m$-nitrobenzyl alcohol matrix) mode. IR data, which were measured as films on $\mathrm{NaCl}$ plates (oils) or as $\mathrm{KBr}$ pellets (solids), are given only when relevant functions $(\mathrm{C}=\mathrm{O}, \mathrm{OH})$ are present. Optical rotations were measured at $25^{\circ} \mathrm{C}$. Reactions which required an inert atmosphere (all except those involving water in the reaction medium) were carried out under dry $\mathrm{N}_{2}$ with flame-dried glassware. Commercial reagents were used as received. THF and $\mathrm{Et}_{2} \mathrm{O}$ were freshly distilled from sodium-benzophenone ketyl. Dichloromethane was freshly distilled from $\mathrm{CaH}_{2}$. Tertiary amines were freshly distilled from $\mathrm{KOH}$. Unless detailed otherwise, "workup" means pouring the reaction mixture into brine, followed by extraction with the solvent indicated in parenthesis. If the reaction medium was acidic (basic), an additional washing with $5 \%$ aq $\mathrm{NaHCO}_{3}\left(\mathrm{aq} \mathrm{NH}_{4} \mathrm{Cl}\right)$ was performed. New washing with brine, drying over anhydrous $\mathrm{Na}_{2} \mathrm{SO}_{4}$ and elimination of the solvent under reduced pressure were followed by chromatography on a silica gel column $(60-200 \mu \mathrm{m})$ with the indicated eluent. Where solutions were filtered through a Celite pad, the pad was additionally rinsed with the same solvent used, and the washing liquids incorporated to the main organic layer.

\section{General reaction scheme}
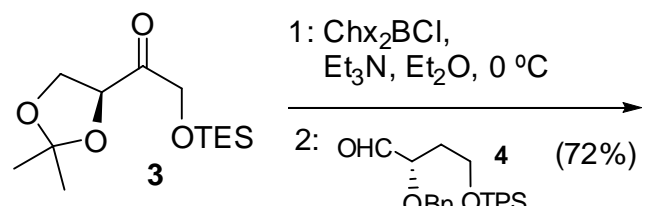

1: $\mathrm{Chx}_{2} \mathrm{BCl}$, $\mathrm{Et}_{3} \mathrm{~N}, \mathrm{Et}_{2} \mathrm{O}, 0^{\circ} \mathrm{C}$

2: $\mathrm{OHC} \underset{(72 \%)}{\longrightarrow} 4$

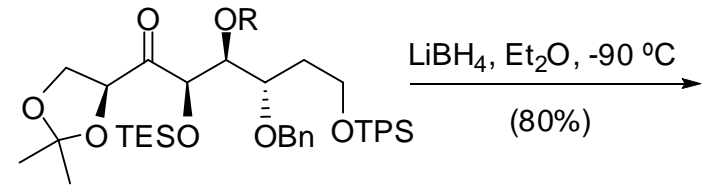

$\begin{array}{ll}5 & \mathrm{R}=\mathrm{H} \\ 6 & \mathrm{R}=\mathrm{SEM} \\ \mathrm{CHMCl}, \mathrm{iPr} & \mathrm{NEt} \\ \mathrm{CH}_{2} \mathrm{Cl}_{2}, \mathrm{rt}, 24 \mathrm{~h}(75 \%)\end{array}$
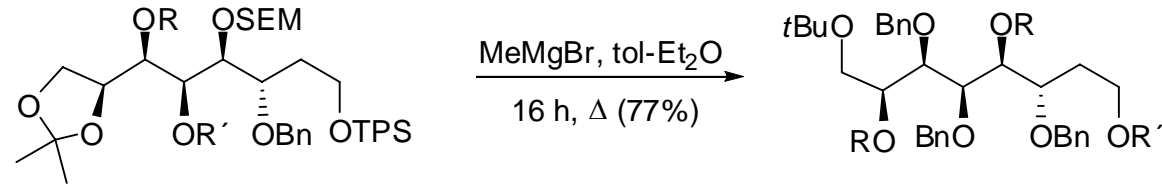

$\underset{80{ }^{\circ} \mathrm{C}, 24 \mathrm{~h}(60 \%}{\stackrel{\mathrm{BnNH}_{2}, \mathrm{Nal}, \mathrm{DMSO}}{\longrightarrow}}$

$7 \mathrm{R}=\mathrm{H} \mathrm{R}^{\prime}=$ TES

$8 R=R^{\prime}=H$

$9 \mathrm{R}=\mathrm{R}^{\prime}=\mathrm{Bn}$

DDQ, aq THF, rt, $24 \mathrm{~h}(74 \%)$

$\mathrm{BnBr}, \mathrm{NaH}, \mathrm{THF}, 4 \mathrm{~h}, 40^{\circ} \mathrm{C}$ (80\%)

$\begin{aligned} & 10 \mathrm{R}=\mathrm{H}^{\prime}=\mathrm{R} \mathrm{R}^{\prime} \\ & 11 \mathrm{R}=\mathrm{R}^{\prime}=\mathrm{H} \\ & 12 \mathrm{R}=\mathrm{R}^{\prime}=\mathrm{Ms}\end{aligned} \mathrm{MsCl}, \mathrm{Et}_{3} \mathrm{~N}, \mathrm{CH}_{2} \mathrm{Cl}_{2}$, rt, 2h

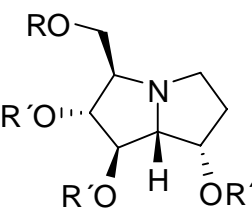

1: TFA, $\mathrm{CH}_{2} \mathrm{Cl}_{2}$, rt, $15 \mathrm{~h}$

2: aq $\mathrm{NH}_{3}(78 \%$ overall from 13)

$\begin{array}{ll}13 & \mathrm{R}=t \mathrm{Bu} \quad \mathrm{R}^{\prime}=\mathrm{Bn} \\ 14 \mathrm{R}=t \mathrm{Bu} & \mathrm{R}^{\prime}=\mathrm{H}\end{array} \square \mathrm{H}_{2} / \mathrm{Pd}(\mathrm{OH})_{2}, \mathrm{rt}, 2 \mathrm{~d}(75 \%)$

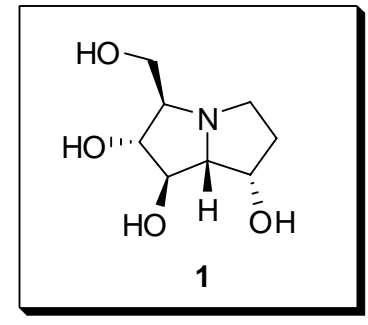




\section{Experimental procedures}

\section{Preparation of aldehyde 4}
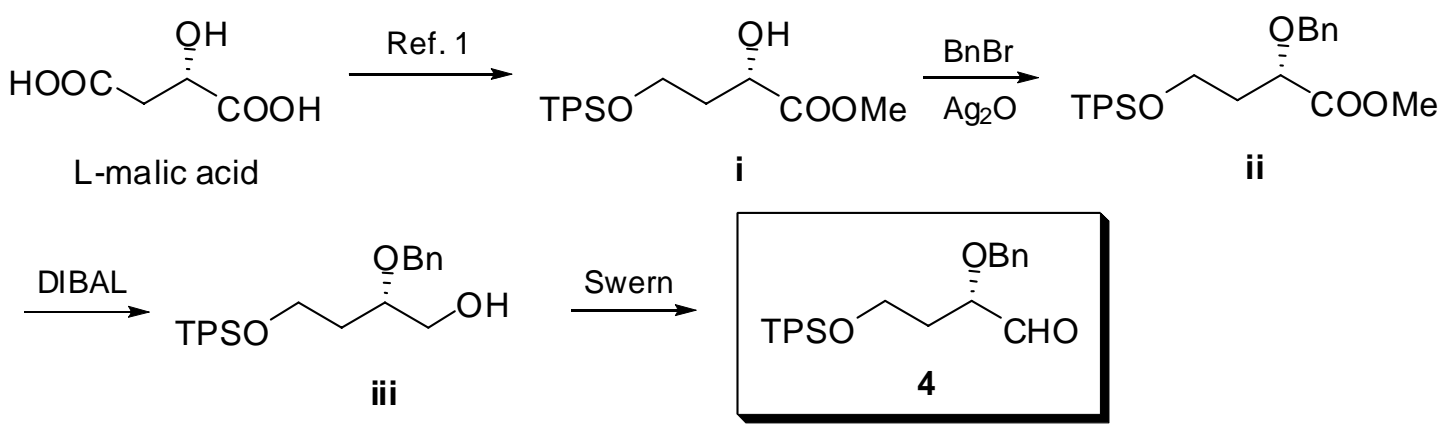

The $\alpha$-hydroxy ester $\mathbf{i}$ was prepared in three steps from L-malic acid ${ }^{1}$ and showed the expected spectral data. ${ }^{2}$ It was used in crude form for the next benzylation step.

${ }^{1}$ Hayashi, Y.; Yamaguchi, J.; Shoji, M. Tetrahedron 2002, 58, 9839-9846.

${ }^{2}$ Hanessian, S.; Tehim, A.; Chen, P. J. Org. Chem. 1993, 58, 7768-7781.

(S)-4-(Tert-Butyldiphenylsilyloxy)-2-benzyloxybutanoic acid methyl ester (ii). Freshly prepared, dried $\mathrm{Ag}_{2} \mathrm{O}$ (8.34 g, $36 \mathrm{mmol}$ ) was suspended under $\mathrm{N}_{2}$ in dry $\mathrm{Et}_{2} \mathrm{O}$ (25 mL). Alcohol i (3.35 g, 9 mmol) disolved in dry $\mathrm{Et}_{2} \mathrm{O}(15 \mathrm{~mL})$ was then added, followed by addition of $\mathrm{BnBr}(4 \mathrm{~mL}$, ca. 36 $\mathrm{mmol}$ ). The reaction mixture was stirred at room temperature in the darkness for $24 \mathrm{~h}$. After filtration through Celite and removal of all volatiles under reduced pressure, the residue was chromatographed on silica gel (hexanes-EtOAc, 95:5) to yield ii (3.83 g, 92\%): colorless oil; $[\alpha]_{\mathrm{D}}-31.7$ (c 1.4; $\left.\mathrm{CHCl}_{3}\right)$; ${ }^{1} \mathrm{H}$ NMR $\delta$ 7.70-7.65 (4H, br d, $\left.J=7 \mathrm{~Hz}\right), 7.45-7.30(11 \mathrm{H}, \mathrm{m}), 4.72(1 \mathrm{H}, \mathrm{d}, J=11.3 \mathrm{~Hz}), 4.43(1 \mathrm{H}, \mathrm{d}, J$ $=11.3 \mathrm{~Hz}), 4.30(1 \mathrm{H}, \mathrm{dd}, J=9,4 \mathrm{~Hz}), 3.88(1 \mathrm{H}, \mathrm{m}), 3.78(1 \mathrm{H}, \mathrm{m}), 3.76(3 \mathrm{H}, \mathrm{s}), 2.08(1 \mathrm{H}, \mathrm{m}), 1.96$ $(1 \mathrm{H}, \mathrm{m}), 1.06(9 \mathrm{H}, \mathrm{s}) ;{ }^{13} \mathrm{C} \mathrm{NMR} \delta 173.5,137.6,133.7,133.6,19.2$ (C), 135.6 (x 2), 135.5 (x 2), 129.6 (x 2), 128.3 (x 2), 127.9 (x 2), 127.7 (x 3), 127.6 (x 2), 75.1 (CH), 72.6, 59.6, $35.9\left(\mathrm{CH}_{2}\right), 51.8,26.9$ (x 3) $\left(\mathrm{CH}_{3}\right)$; IR $v_{\max } 1751(\mathrm{C}=\mathrm{O}) \mathrm{cm}^{-1}$; HR EIMS m/z (\%rel. int.) $405.1529\left(\mathrm{M}^{+}-t \mathrm{Bu}, 1\right), 237$ (32), 209 (66), 91 (100). Calcd. for $\mathrm{C}_{28} \mathrm{H}_{34} \mathrm{O}_{4} \mathrm{Si}-t \mathrm{Bu}, 405.1522$.

(S)-4-(Tert-Butyldiphenylsilyloxy)-2-benzyloxy-1-butanol (iii). An ice-cooled solution of ester ii (3.7 $\mathrm{g}, 8 \mathrm{mmol})$ in dry hexane $(10 \mathrm{~mL})$ was treated dropwise under $\mathrm{N}_{2}$ with DIBAL (1M solution in hexane, $20 \mathrm{~mL}, 20 \mathrm{mmol}$ ). The mixture was stirred at $0{ }^{\circ} \mathrm{C}$ for $2.5 \mathrm{~h}$ and then quenched through addition of aq satd $\mathrm{NH}_{4} \mathrm{Cl}(1.5 \mathrm{~mL})$. The stirring was continued at room temperature for $30 \mathrm{~min}$. Filtration through Celite (washing with EtOAc) and removal of all volatiles under reduced pressure gave a residue which was chromatographed on silica gel (hexanes-EtOAc, 80:20). This provided primary alcohol iii (3.13 g, 90\%): colorless oil; $[\alpha]_{\mathrm{D}}+4.2\left(c\right.$ 3.1; $\left.\mathrm{CHCl}_{3}\right) ;{ }^{1} \mathrm{H} \mathrm{NMR} \delta 7.75-7.70(4 \mathrm{H}$, br d, $J=7 \mathrm{~Hz}), 7.50-7.30$ $(11 \mathrm{H}$, br m), $4.59(2 \mathrm{H}, \mathrm{AB}$ system, $J=11.5 \mathrm{~Hz}), 3.90-3.80(4 \mathrm{H}, \mathrm{m}), 3.62(1 \mathrm{H}, \mathrm{dd}, J=11.3,5.3 \mathrm{~Hz})$, $2.50\left(1 \mathrm{H}\right.$, br s, OH), $1.95(1 \mathrm{H}, \mathrm{m}), 1.86(1 \mathrm{H}, \mathrm{m}), 1.14(9 \mathrm{H}, \mathrm{s}) ;{ }^{13} \mathrm{C}$ NMR $\delta 138.5,133.6,133.5,19.1$ 
(C), 135.5 (x 4), 129.6 (x 2), 128.3 (x 2), 127.7 (x 5), 127.6 (x 2), $77.1(\mathrm{CH}), 71.6,64.3,60.3,34.1$ $\left(\mathrm{CH}_{2}\right), 26.9$ (x 3) $\left(\mathrm{CH}_{3}\right)$; IR $v_{\max } 3440(\mathrm{br}, \mathrm{OH}) \mathrm{cm}^{-1}$; HR FABMS m/z $435.2345\left(\mathrm{M}+\mathrm{H}^{+}\right)$. Calcd. for $\mathrm{C}_{27} \mathrm{H}_{35} \mathrm{O}_{3} \mathrm{Si}, 435.2355$.

(S)-4-(Tert-Butyldiphenylsilyloxy)-2-benzyloxy-1-butanal (4). Oxalyl chloride (790 $\mu \mathrm{L}$, ca. 9 mmol) was dissolved under $\mathrm{N}_{2}$ in dry $\mathrm{CH}_{2} \mathrm{Cl}_{2}(30 \mathrm{~mL})$ and cooled to $-78^{\circ}$. Dry DMSO (700 $\mu \mathrm{L}$, ca. $\left.10 \mathrm{mmol}\right)$ was then added dropwise, followed by stirring for $5 \mathrm{~min}$. Alcohol iii $(2.6 \mathrm{~g}, 6 \mathrm{mmol})$ was dissolved in dry $\mathrm{CH}_{2} \mathrm{Cl}_{2}(18 \mathrm{~mL})$ and added via syringe to the reaction mixture, with stirring for $15 \mathrm{~min}$. at the same temperature. After addition of $\mathrm{Et}_{3} \mathrm{~N}(3.8 \mathrm{~mL}$, ca. $27 \mathrm{mmol}$ ), the stirring was continued for $15 \mathrm{~min}$. a $-78^{\circ}$ and then for $30 \mathrm{~min}$. at $0{ }^{\circ} \mathrm{C}$. Work-up (extraction with $\mathrm{CH}_{2} \mathrm{Cl}_{2}$ ) gave an oily residue, which was used as such in the next reaction: ${ }^{1} \mathrm{H}$ NMR $\delta 9.76(1 \mathrm{H}, \mathrm{d}, J=1.5 \mathrm{~Hz}), 7.70-7.65(4 \mathrm{H}, \mathrm{m}), 7.45-7.30$ $(11 \mathrm{H}, \mathrm{br} \mathrm{m}), 4.70(1 \mathrm{H}, \mathrm{d}, J=11.5 \mathrm{~Hz}), 4.56(1 \mathrm{H}, \mathrm{d}, J=11.5 \mathrm{~Hz}), 4.08(1 \mathrm{H}, \mathrm{ddd}, J=7,5,1.5 \mathrm{~Hz}), 3.84$ (1H, ddd, $J=10.5,6.2,5 \mathrm{~Hz}), 3.80(1 \mathrm{H}, \mathrm{ddd}, J=10.5,7.2,5 \mathrm{~Hz}), 2.05-1.90(2 \mathrm{H}, \mathrm{br} \mathrm{m}), 1.04(9 \mathrm{H}, \mathrm{s})$; ${ }^{13} \mathrm{C}$ NMR $\delta$ 137.5, 133.6, 133.5, 19.2 (C), 203.5, 135.6 (x 4), 129.7 (x 2), 128.5 (x 2), 128.0, 127.9 (x 2), $127.7(\mathrm{x} 4), 80.8(\mathrm{CH}), 72.7,59.1,33.6\left(\mathrm{CH}_{2}\right), 26.9(\mathrm{x} 3)\left(\mathrm{CH}_{3}\right)$; IR $v_{\max } 1733(\mathrm{C}=\mathrm{O}) \mathrm{cm}^{-1}$.

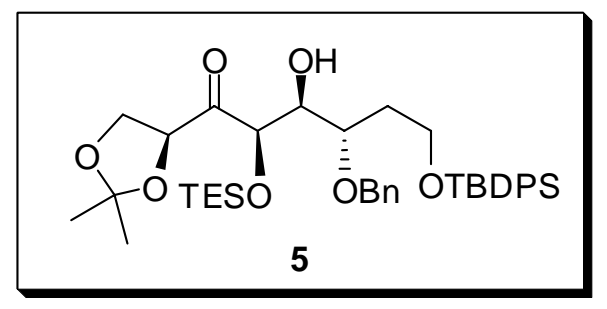

(2S,4R,5S,6S)-6-O-Benzyl-8-O-(tert-butyldiphenylsilyl)-1,2-di-O-isopropylidene-4-O(triethylsilyl)-1,2,4,5,6,8-hexahydroxyoctane-3-one (5). $\mathrm{Chx}_{2} \mathrm{BCl}$ (neat, $790 \mu \mathrm{L}$, ca. $3.6 \mathrm{mmol}$ ) was added under $\mathrm{N}_{2}$ via syringe to an ice-cooled solution of $\mathrm{Et}_{3} \mathrm{~N}(560 \mu \mathrm{L}, 4 \mathrm{mmol})$ in anhydrous $\mathrm{Et}_{2} \mathrm{O}(10$ $\mathrm{mL}$ ). Ketone 3 (548 $\mathrm{mg}, 2 \mathrm{mmol}$ ) was dissolved in anhydrous ether $(10 \mathrm{~mL})$ and added dropwise via syringe to the reagent solution. The reaction mixture was then stirred at $0{ }^{\circ} \mathrm{C}$ for $1 \mathrm{~h}$. After addition of a solution of freshly prepared aldehyde $4(2.6 \mathrm{~g}$, ca. $6 \mathrm{mmol})$ in ether $(10 \mathrm{~mL})$, the reaction mixture was stirred at the same temperature for $5 \mathrm{~h}$. Then phosphate buffer solution $(\mathrm{pH} 7,12 \mathrm{~mL})$ and $\mathrm{MeOH}(12$ $\mathrm{mL}$ ) were added, followed by $30 \%$ aq $\mathrm{H}_{2} \mathrm{O}_{2}$ solution $(6 \mathrm{~mL})$. After stirring for $1 \mathrm{~h}$ at room temperature, the mixture was worked up (extraction with $\mathrm{Et}_{2} \mathrm{O}$ ). Solvent removal in vacuo and column chromatography of the residue on silica gel (hexanes-EtOAc, 95:5) afforded 5 (1.017 g, 72\%): colorless oil; $[\alpha]_{\mathrm{D}}-33.2$ (c 1.16; $\left.\mathrm{CHCl}_{3}\right) ;{ }^{1} \mathrm{H}$ NMR $\delta 7.68(4 \mathrm{H}, \mathrm{m}), 7.45-7.25(11 \mathrm{H}, \mathrm{m}), 5.13(1 \mathrm{H}, \mathrm{d}, J=$ $1.5 \mathrm{~Hz}), 4.61(1 \mathrm{H}, \mathrm{d}, J=11.3 \mathrm{~Hz}), 4.58(1 \mathrm{H}, \mathrm{dd}, J=7.5,5.5 \mathrm{~Hz}), 4.46(1 \mathrm{H}, \mathrm{d}, J=11.3 \mathrm{~Hz}), 4.20-4.10$ $(3 \mathrm{H}, \mathrm{m}), 3.90-3.80(3 \mathrm{H}, \mathrm{m}), 2.68(1 \mathrm{H}, \mathrm{d}, J=10 \mathrm{~Hz}, \mathrm{OH}), 2.11(1 \mathrm{H}, \mathrm{m}), 1.98(1 \mathrm{H}, \mathrm{m}), 1.35(3 \mathrm{H}, \mathrm{s}), 1.31$ $(3 \mathrm{H}, \mathrm{s}), 1.06(9 \mathrm{H}, \mathrm{s}), 0.95(9 \mathrm{H}, \mathrm{t}, J=8 \mathrm{~Hz}), 0.63(6 \mathrm{H}, \mathrm{m}) ;{ }^{13} \mathrm{C}$ NMR $\delta 208.5,138.6,133.6$ (x 2), 110.8, 19.1 (C), 135.6 (x 4), 129.6, 128.2 (x 2), 127.7 (x 2), 127.6 (x 3), 127.4, 127.3 (x 2), 78.5, 76.4, 76.3, $73.1(\mathrm{CH}), 71.2,66.6,60.3,33.1,5.1$ (x 3) $\left(\mathrm{CH}_{2}\right), 26.9$ (x 3), 25.9, 24.9, 6.9 (x 3) $\left(\mathrm{CH}_{3}\right) . \mathrm{IR} v_{\max } 3480$ 
(br, OH), $1732(\mathrm{C}=\mathrm{O}) \mathrm{cm}^{-1}$. HR FABMS m/z $707.3748\left(\mathrm{M}+\mathrm{H}^{+}\right)$. Calcd. for $\mathrm{C}_{40} \mathrm{H}_{59} \mathrm{O}_{7} \mathrm{Si}_{2}, 707.3799$. Anal. Calcd. for $\mathrm{C}_{40} \mathrm{H}_{58} \mathrm{O}_{7} \mathrm{Si}_{2}$ : C, 67.95; H, 8.27. Found: C, 68.01; H, 8.40.

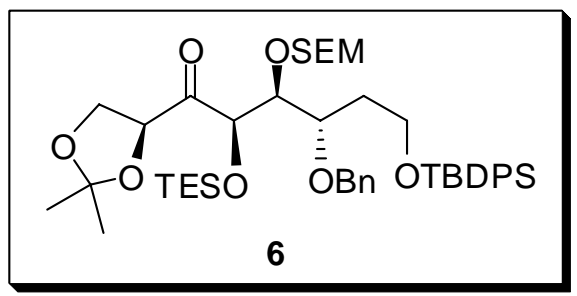

(2S,4R,5S,6S)-6-O-Benzyl-8-O-(tert-butyldiphenylsilyl)-1,2-di-O-isopropylidene-4-O-

(triethylsilyl)-5-O-(trimethylsilylethoxymethyl)-1,2,4,5,6,8-hexahydroxyoctane-3-one (6). Aldol 5 (990 mg, $1.4 \mathrm{mmol})$ was dissolved under $\mathrm{N}_{2}$ in dry $\mathrm{CH}_{2} \mathrm{Cl}_{2}(6 \mathrm{~mL})$ and treated at room temperature with SEM chloride $(990 \mu \mathrm{L}, 5.6 \mathrm{mmol})$, DMAP $(12 \mathrm{mg}, 0.1 \mathrm{mmol})$ and $i \mathrm{Pr}_{2} \mathrm{NEt}(1.2 \mathrm{~mL}, 7 \mathrm{mmol})$. The reaction mixture was stirred at room temperature for $24 \mathrm{~h}$. Work-up (extraction with $\mathrm{CH}_{2} \mathrm{Cl}_{2}$ ) and column chromatography on silica gel (hexanes-EtOAc, 95:5) afforded 6 (879 mg, 75\%): colorless oil; $[\alpha]_{\mathrm{D}}-29.4$ (c 1.7; $\left.\mathrm{CHCl}_{3}\right) ;{ }^{1} \mathrm{H}$ NMR $\delta$ 7.70-7.65 (4H, m), 7.40-7.20 (11H, m), $4.95(1 \mathrm{H}, \mathrm{d}, J=3.3 \mathrm{~Hz})$, $4.83(1 \mathrm{H}$, br dd, $J=8,6 \mathrm{~Hz}), 4.75(2 \mathrm{H}, \mathrm{AB}$ system, $J=7 \mathrm{~Hz}), 4.58(1 \mathrm{H}, \mathrm{d}, J=11.3 \mathrm{~Hz}), 4.52(1 \mathrm{H}, \mathrm{d}, J$ $=11.3 \mathrm{~Hz}), 4.22(1 \mathrm{H}, \mathrm{dd}, J=4.6,3.3 \mathrm{~Hz}), 4.11(1 \mathrm{H}, \mathrm{dd}, J=8.5,6 \mathrm{~Hz}), 4.00(1 \mathrm{H}, \mathrm{dd}, J=8.5,8 \mathrm{~Hz})$, $3.96(1 \mathrm{H}, \mathrm{m}), 3.82(2 \mathrm{H}, \mathrm{m}), 3.63(1 \mathrm{H}, \mathrm{dt}, J=10,6.5 \mathrm{~Hz}), 3.51(1 \mathrm{H}, \mathrm{dt}, J=10,6.5 \mathrm{~Hz}), 2.00(1 \mathrm{H}, \mathrm{m})$, $1.90(1 \mathrm{H}, \mathrm{m}), 1.36(3 \mathrm{H}, \mathrm{s}), 1.33(3 \mathrm{H}, \mathrm{s}), 1.06(9 \mathrm{H}, \mathrm{s}), 0.97(9 \mathrm{H}, \mathrm{t}, J=8 \mathrm{~Hz}), 0.85(2 \mathrm{H}, \mathrm{m}), 0.63(6 \mathrm{H}, \mathrm{q}$, $J=8 \mathrm{~Hz}$ ), $0.01(9 \mathrm{H}, \mathrm{s}) ;{ }^{13} \mathrm{C}$ NMR $\delta 207.2,138.5,133.8$ (x 2), 110.5, 19.1 (C), 135.6 (x 4), 129.5 (x 2), 128.2 (x 2), 127.6 (x 4), 127.5 (x 2), 127.4, 80.1, 78.3, 76.8, 76.2 (CH), 95.5, 72.1, 66.0, 65.9, 60.6, 33.6, 18.0, 5.1 (x 3) $\left(\mathrm{CH}_{2}\right), 26.9$ (x 3), 26.0, 25.2, 6.9 (x 3), -1.5 (x 3) $\left(\mathrm{CH}_{3}\right)$; IR v $v_{\max } 1732(\mathrm{C}=\mathrm{O}) \mathrm{cm}^{-1}$; HR EIMS m/z (\% rel. int.) $836.4557\left(\mathrm{M}^{+}, 1\right), 821\left(\mathrm{M}^{+}-\mathrm{Me}, 1\right), 807\left(\mathrm{M}^{+}-\mathrm{Et}, 1\right), 346$ (22), 91 (100). Calcd. para $\mathrm{C}_{46} \mathrm{H}_{72} \mathrm{O}_{8} \mathrm{Si}_{3}$, 836.4535. Anal. Calcd. for $\mathrm{C}_{46} \mathrm{H}_{72} \mathrm{O}_{8} \mathrm{Si}_{3}$ : C, 65.98; H, 8.67. Found: C, 66.06; $\mathrm{H}, 8.51$.

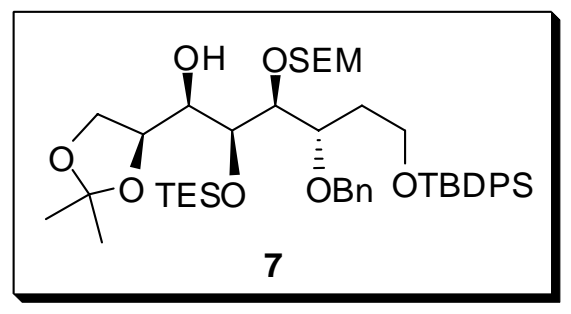

(1R,2S,3S,4S)-4-(Benzyloxy)-6-(tert-butyldiphenylsilyloxy)-1-[(S)-2,2-dimethyl-1,3-dioxolan-4-yl]2-(triethylsilyloxy)-3-(2-trimethylsilylethoxymethoxy)hexan-1-ol (7). A solution of compound 6 $(837 \mathrm{mg}, 1 \mathrm{mmol})$ in dry $\mathrm{Et}_{2} \mathrm{O}(3 \mathrm{~mL})$ was cooled to $-90{ }^{\circ} \mathrm{C}$. Then, a solution of $\mathrm{LiBH}_{4}\left(2 \mathrm{M}\right.$ in $\mathrm{Et}_{2} \mathrm{O}, 1$ $\mathrm{mL}, 2 \mathrm{mmol}$ ) was added dropwise under $\mathrm{N}_{2}$. The reaction mixture was stirred for $2 \mathrm{~h}$ at $-90^{\circ} \mathrm{C}$. Workup (extraction with $\mathrm{Et}_{2} \mathrm{O}$ ) and column chromatography on silica gel (hexanes-EtOAc, 90:10) provided 
compound 7 (671 mg, 80\%): colorless oil; $[\alpha]_{\mathrm{D}}-25.3$ (c 2.5; $\mathrm{CHCl}_{3}$ ); ${ }^{1} \mathrm{H} \mathrm{NMR} \delta$ 7.70-7.65 (4H, m), 7.45-7.25 (11H, m), $4.80(1 \mathrm{H}, \mathrm{d}, J=6.5 \mathrm{~Hz}), 4.76(1 \mathrm{H}, \mathrm{d}, J=6.5 \mathrm{~Hz}), 4.69(1 \mathrm{H}, \mathrm{d}, J=11.3 \mathrm{~Hz}), 4.45$ $(1 \mathrm{H}, \mathrm{d}, J=11.3 \mathrm{~Hz}), 4.18(1 \mathrm{H}$, br q, J 6.5 Hz), 4.00-3.95 (2H, m), $3.94(1 \mathrm{H}, \mathrm{m}), 3.90(1 \mathrm{H}, \mathrm{m}), 3.83$ $(1 \mathrm{H}, \mathrm{m}), 3.80-3.70(2 \mathrm{H}, \mathrm{m}), 3.70-3.60(3 \mathrm{H}, \mathrm{m}), 2.70(1 \mathrm{H}, \mathrm{d}, J=9 \mathrm{~Hz}, \mathrm{OH}), 2.00-1.90(2 \mathrm{H}, \mathrm{m}), 1.43$ $(3 \mathrm{H}, \mathrm{s}), 1.39(3 \mathrm{H}, \mathrm{s}), 1.07(9 \mathrm{H}, \mathrm{s}), 1.02(9 \mathrm{H}, \mathrm{t}, J=8 \mathrm{~Hz}), 0.89(2 \mathrm{H}, \mathrm{m}), 0.69(6 \mathrm{H}, \mathrm{q}, J=8 \mathrm{~Hz}), 0.00$ $(9 \mathrm{H}, \mathrm{s}) ;{ }^{13} \mathrm{C}$ NMR $\delta$ 138.7, 134.0, 133.9, 109.5, 19.2 (C), 135.6 (x 4), 129.5 (x 2), 128.2 (x 2), 127.6 (x 4), 127.5 (x 2), 127.4, 78.6, 77.5, 76.3, 73.5, $70.0(\mathrm{CH}), 95.6,71.9,66.3,65.5,60.7,33.7,18.0,5.2$ (x 3) $\left(\mathrm{CH}_{2}\right), 26.9$ (x 3), 26.5, 25.7, 7.0 (x 3), -1.5 (x 3) $\left(\mathrm{CH}_{3}\right)$; IR $v_{\max } 3550(\mathrm{br}, \mathrm{OH}) \mathrm{cm}^{-1}$; HR EIMS m/z (\% rel. int.) $823.4475\left(\mathrm{M}^{+}-\mathrm{Me}, 1\right), 255$ (28), 101 (45), 91 (100). Calcd. para $\mathrm{C}_{46} \mathrm{H}_{74} \mathrm{O}_{8} \mathrm{Si}_{3}-\mathrm{Me}_{\text {, }}$ 823.4457. Anal. Calcd. for $\mathrm{C}_{46} \mathrm{H}_{74} \mathrm{O}_{8} \mathrm{Si}_{3}$ : C, 65.83; H, 8.89. Found: C, 66.00; H, 8.81.

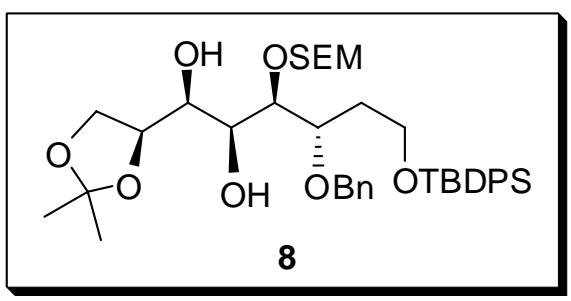

(1S,2S,3R,4S)-4-(Benzyloxy)-6-(tert-butyldiphenylsilyloxy)-1-[(S)-2,2-dimethyl-1,3-dioxolan-4-yl]3-[(2-(trimethylsilylethoxy)methoxy]hexane-1,2-diol (8). Compound 7 (630 mg, $0.75 \mathrm{mmol})$ was dissolved in a 9:1 THF- $\mathrm{H}_{2} \mathrm{O}$ mixture $(5 \mathrm{~mL})$ and treated dropwise with a solution of DDQ (17 mg, $0.075 \mathrm{mmol}, 0.1$ equiv) in the same solvent mixture $(3 \mathrm{~mL})$. The reaction mixture was stirred at room temperature for $24 \mathrm{~h}$. Caution: it is important to avoid an excess of DDQ and to keep the indicated reaction time in order to prevent a partial cleavage of the acetal group. Work-up (extraction with EtOAc) and column chromatography on silica gel (hexanes-EtOAc, 70:30) furnished recovered 7 (63 $\mathrm{mg}, 10 \%)$ and 8 (402 mg, 82\% based on recovered 7): colorless oil; $[\alpha]_{\mathrm{D}}+1.1\left(c \quad 1.95 ; \mathrm{CHCl}_{3}\right) ;{ }^{1} \mathrm{H}$ NMR $\delta$ 7.70-7.65 (4H, m), 7.45-7.25 $(11 \mathrm{H}, \mathrm{m}), 4.80(1 \mathrm{H}, \mathrm{d}, J=6.5 \mathrm{~Hz}), 4.73(1 \mathrm{H}, \mathrm{d}, J=6.5 \mathrm{~Hz}), 4.66$ $(1 \mathrm{H}, \mathrm{d}, J=11.3 \mathrm{~Hz}), 4.50(1 \mathrm{H}, \mathrm{d}, J=11.3 \mathrm{~Hz}), 4.32(1 \mathrm{H}$, br q, $J=6.5 \mathrm{~Hz}), 4.00(2 \mathrm{H}, \mathrm{m}), 3.90(1 \mathrm{H}, \mathrm{dd}$, $J=5.3,3.1 \mathrm{~Hz}), 3.85(1 \mathrm{H}, \mathrm{m}), 3.80-3.70(3 \mathrm{H}, \mathrm{m}), 3.65-3.55(3 \mathrm{H}, \mathrm{m}), 2.90(1 \mathrm{H}, \mathrm{d}, J=6 \mathrm{~Hz}, \mathrm{OH}), 1.90-$ $1.80(2 \mathrm{H}, \mathrm{m}), 1.41(3 \mathrm{H}, \mathrm{s}), 1.37(3 \mathrm{H}, \mathrm{s}), 1.05(9 \mathrm{H}, \mathrm{s}), 0.95(2 \mathrm{H}, \mathrm{m}), 0.01(9 \mathrm{H}, \mathrm{s})$ (one hydroxyl signal not detected); ${ }^{13} \mathrm{C}$ NMR $\delta$ 138.2, 133.7 (x 2), 109.6, 19.2 (C), 135.6 (x 2), 135.5 (x 2), 129.6 (x 2), 128.4 (x 2), 127.9 (x 2), 127.7 (x 5), 81.1, 77.2, 76.4, 71.6, 71.2 (CH), 96.3, 72.6, 66.1, 66.0, 60.2, 33.7, $18.1\left(\mathrm{CH}_{2}\right), 26.9$ (x 3), 26.5, 25.5, -1.5 (x 3) $\left(\mathrm{CH}_{3}\right)$; IR $v_{\max } 3470(\mathrm{br}, \mathrm{OH}) \mathrm{cm}^{-1}$; HR EIMS m/z (\% rel. int.) $709.3582\left(\mathrm{M}^{+}-\mathrm{Me}, 1\right), 323$ (3), 255 (14), 235 (11.7), 199 (12), 91 (100). Calcd. para $\mathrm{C}_{40} \mathrm{H}_{60} \mathrm{O}_{8} \mathrm{Si}_{2}-\mathrm{Me}, 709.3592$. Anal. Calcd. for $\mathrm{C}_{40} \mathrm{H}_{60} \mathrm{O}_{8} \mathrm{Si}_{2}: \mathrm{C}, 66.26 ; \mathrm{H}$, 8.34. Found: C, 66.20; H, 8.51. 


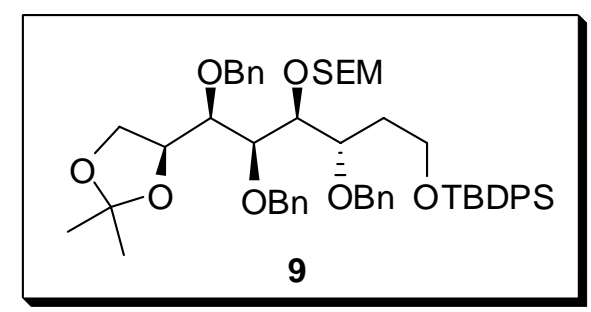

(4S)-[(1R,2S,3S,4S)-1,2,4-Tris(benzyloxy)-3-(2-trimethylsilylethoxymethoxy)-6-(tert-

butyldiphenylsilyloxy)hexyl]-2,2-dimethyl-1,3-dioxolane (9). A solution of compound 8 (362 mg, ca. $0.5 \mathrm{mmol})$ in dry THF $(5 \mathrm{~mL})$ was added dropwise under $\mathrm{N}_{2}$ to an ice-cooled suspension of $\mathrm{NaH}(95 \%$ dry powder, $40 \mathrm{mg}, 1.6 \mathrm{mmol})$ in dry THF $(3 \mathrm{~mL})$. After stirring for $30 \mathrm{~min}$. at $0{ }^{\circ} \mathrm{C}$, TBAI $(6 \mathrm{mg}$, $0.015 \mathrm{mmol})$ and $\mathrm{BnBr}(178 \mu \mathrm{L}, 1.5 \mathrm{mmol})$ were sequentially added. The mixture was stirred for 15 min. at room temperature and then for further $4 \mathrm{~h}$ at $40{ }^{\circ} \mathrm{C}$. Work-up (extraction with EtOAc) and column chromatography on silica gel (hexanes-EtOAc, 90:10) yielded 9 (362 mg, 80\%): colorless oil; $[\alpha]_{\mathrm{D}}-35.9\left(\mathrm{c} 1.1 ; \mathrm{CHCl}_{3}\right) ;{ }^{1} \mathrm{H}$ NMR $\delta$ 7.65-7.60 $(4 \mathrm{H}, \mathrm{m}), 7.40-7.15(21 \mathrm{H}, \mathrm{m}), 4.83(2 \mathrm{H}, \mathrm{s}), 4.76(1 \mathrm{H}, \mathrm{d}$, $J=11.5 \mathrm{~Hz}), 4.74(1 \mathrm{H}, \mathrm{d}, J=11.8 \mathrm{~Hz}), 4.64(1 \mathrm{H}, \mathrm{d}, J=11.8 \mathrm{~Hz}), 4.54(2 \mathrm{H}, \mathrm{d}, J=11.5 \mathrm{~Hz}), 4.40(1 \mathrm{H}$, $\mathrm{dt}, J=7.5,6 \mathrm{~Hz}), 4.26(1 \mathrm{H}, \mathrm{d}, J=11.5 \mathrm{~Hz}), 4.20(1 \mathrm{H}, \mathrm{dd}, J=6.2,2.4 \mathrm{~Hz}), 3.87(1 \mathrm{H}, \mathrm{dt}, J=9,2.8 \mathrm{~Hz})$, $3.80(2 \mathrm{H}, \mathrm{m}), 3.70-3.50(5 \mathrm{H}, \mathrm{br} \mathrm{m}), 3.48(1 \mathrm{H}, \mathrm{dd}, J=6,4.3 \mathrm{~Hz}), 1.85(2 \mathrm{H}, \mathrm{m}), 1.36(3 \mathrm{H}, \mathrm{s}), 1.31(3 \mathrm{H}$, s), $1.04(9 \mathrm{H}, \mathrm{s}), 0.82(2 \mathrm{H}, \mathrm{m}),-0.08(9 \mathrm{H}, \mathrm{s}) ;{ }^{13} \mathrm{C} \mathrm{NMR} \delta 138.8,138.5,138.3,133.9,133.8,109.1,19.2$ (C), 135.6 (x 4), 129.6 (x 2), 129.5 (x 2), 128.3 (x 2), 128.2 (x 2), 128.1 (x 2), 128.0 (x 2), 127.6 (x 3), 127.5 (x 2), 127.4 (x 2), 127.2 (x 2), 79.2, 78.8, 77.3, 77.1, 76.8 (CH), 95.9, 74.1, 73.7, 71.6, 66.0, 65.7, 60.7, 33.6, $18.0\left(\mathrm{CH}_{2}\right), 27.0$ (x 3), 26.6, 25.87, -1.5 (x 3) $\left(\mathrm{CH}_{3}\right)$; HR FABMS m/z 927.4647 (M+Na $\left.{ }^{+}\right)$. Calcd. for $\mathrm{C}_{54} \mathrm{H}_{72} \mathrm{O}_{8} \mathrm{Si}_{2}+\mathrm{Na}^{+}$, 927.4663. Anal. Calcd. for $\mathrm{C}_{54} \mathrm{H}_{72} \mathrm{O}_{8} \mathrm{Si}_{2}$ : C, 71.64; H, 8.02. Found: C, $71.80 ; \mathrm{H}, 8.11$.

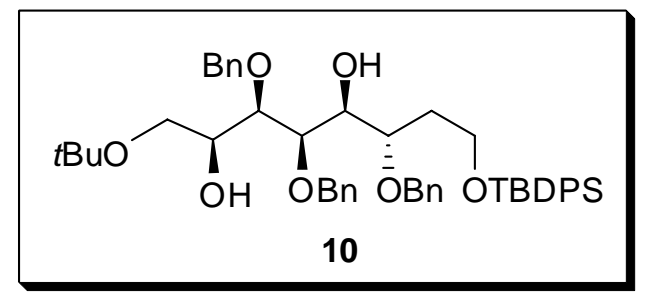

(2S,3R,4R,5S,6S)-3,4,6-Tris(benzyloxy)-1-tert-butoxy-8-(tert-butyldiphenylsilyloxy)octane-2,5-diol (10). Compound $9(317 \mathrm{mg}, 0.35 \mathrm{mmol})$ was dissolved in a 5:1 toluene-Et ${ }_{2} \mathrm{O}$ mixture $(12 \mathrm{~mL})$ and treated with $\mathrm{MeMgBr}\left(3 \mathrm{M}\right.$ solution in $\left.\mathrm{Et}_{2} \mathrm{O}, 700 \mu \mathrm{L}, 2.1 \mathrm{mmol}\right)$. The mixture was then heated at reflux for $16 \mathrm{~h}$. Work-up (extraction with EtOAc) and column chromatography on silica gel (hexanes-EtOAc, 80:20) afforded diol 10 (213 mg, 77\%): colorless oil; $[\alpha]_{\mathrm{D}}+23.2$ (c 1.9; $\left.\mathrm{CHCl}_{3}\right) ;{ }^{1} \mathrm{H}$ NMR $\delta 7.76(4 \mathrm{H}$, br d, $J=7.3 \mathrm{~Hz}), 7.50-7.25(21 \mathrm{H}$, br m), $4.73(1 \mathrm{H}, \mathrm{d}, J=11.5 \mathrm{~Hz}), 4.69(1 \mathrm{H}, \mathrm{d}, J=11.3 \mathrm{~Hz}), 4.61(1 \mathrm{H}$, d, $J=11.5 \mathrm{~Hz}), 4.58(1 \mathrm{H}, \mathrm{d}, J=11.5 \mathrm{~Hz}), 4.51(1 \mathrm{H}, \mathrm{d}, J=11.5 \mathrm{~Hz}), 4.30(1 \mathrm{H}, \mathrm{d}, J=11.5 \mathrm{~Hz}), 4.05$ $(1 \mathrm{H}$, br t, $J=6 \mathrm{~Hz}), 4.02(1 \mathrm{H}$, br d, $J=6.5 \mathrm{~Hz}), 4.00-3.90(3 \mathrm{H}, \mathrm{m}), 3.90-3.85(2 \mathrm{H}, \mathrm{m}), 3.60(1 \mathrm{H}$, br s, 
$\mathrm{OH}), 3.49(1 \mathrm{H}$, br t, $J=7.5 \mathrm{~Hz}), 3.40(1 \mathrm{H}$, br s, OH), $3.32(1 \mathrm{H}, \mathrm{t}, J=7.5 \mathrm{~Hz}), 2.15(1 \mathrm{H}, \mathrm{m}), 2.03(1 \mathrm{H}$, m), $1.23(9 \mathrm{H}, \mathrm{s}), 1.15(9 \mathrm{H}, \mathrm{s}) ;{ }^{13} \mathrm{C}$ NMR $\delta 138.6,138.1,138.0,133.6,133.5,72.9,19.1(\mathrm{C}), 135.5$ (x 4), 129.5 (x 2), 128.3 (x 2), 128.2 (x 4), 128.1 (x 2), 127.7, 127.6, 127.5 (x 4), 127.4 (x 4), 127.3, 76.7, 75.8, 75.4, 70.5, $68.6(\mathrm{CH}), 73.6,73.2,71.3,62.8,60.4,33.4\left(\mathrm{CH}_{2}\right), 27.5$ (x 3), 26.9 (x 3) $\left(\mathrm{CH}_{3}\right)$; IR $v_{\max } 3410(\mathrm{br}, \mathrm{OH}) \mathrm{cm}^{-1}$; HR FABMS m/z $791.4378\left(\mathrm{M}+\mathrm{H}^{+}\right)$. Calcd. para $\mathrm{C}_{49} \mathrm{H}_{63} \mathrm{O}_{7} \mathrm{Si}, 791.4343$. Anal. Calcd. for $\mathrm{C}_{49} \mathrm{H}_{62} \mathrm{O}_{7} \mathrm{Si}: \mathrm{C}, 74.39 ; \mathrm{H}, 7.90$. Found: C, 74.50; H, 8.00.

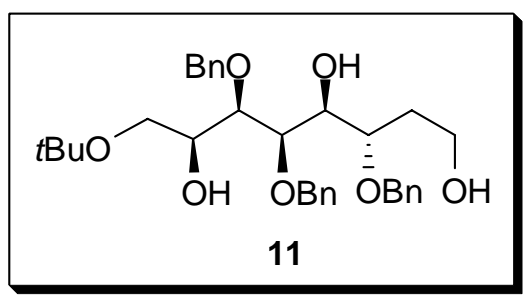

$(3 S, 4 S, 5 R, 6 R, 7 S)-3,5,6$-Tris(benzyloxy)-8-tert-butoxyoctane-1,4,7-triol (11). Compound 10 (198 $\mathrm{mg}, 0.25 \mathrm{mmol})$ was dissolved under $\mathrm{N}_{2}$ in dry THF $(1 \mathrm{~mL})$ and treated with TBAF (100 mg, 0.4 $\mathrm{mmol})$. The reaction mixture was then stirred at room temperature for $1 \mathrm{~h}$. After removal of all volatiles in vacuo, the oily residue was purified by column chromatography on silica gel (hexanes-EtOAc, 1:1) to give triol 11 (134 mg, 97\%): colorless oil; $[\alpha]_{\mathrm{D}}+32.0$ (c 2.2; $\left.\mathrm{CHCl}_{3}\right) ;{ }^{1} \mathrm{H} \mathrm{NMR} \delta$ 7.35-7.20 (15H, m), $4.63(1 \mathrm{H}, \mathrm{d}, J=11.5 \mathrm{~Hz}), 4.58(1 \mathrm{H}, \mathrm{d}, J=11.5 \mathrm{~Hz}), 4.54(1 \mathrm{H}, \mathrm{d}, J=11.5 \mathrm{~Hz}), 4.52(1 \mathrm{H}, \mathrm{d}, J=11.5$ $\mathrm{Hz}), 4.40(1 \mathrm{H}, \mathrm{d}, J=11.5 \mathrm{~Hz}), 4.25(1 \mathrm{H}, \mathrm{d}, J=11.5 \mathrm{~Hz}), 4.10(1 \mathrm{H}, \mathrm{br}$ s, OH), $4.00(1 \mathrm{H}, \mathrm{m}), 3.95(2 \mathrm{H}$, $\mathrm{d}, J=6.5 \mathrm{~Hz}+$ br s, OH), $3.80(2 \mathrm{H}, \mathrm{m}), 3.75(1 \mathrm{H}, \mathrm{d}, J=6.5 \mathrm{~Hz}), 3.70(1 \mathrm{H}, \mathrm{m}), 3.40(1 \mathrm{H}$, br t,$J \sim 7.7$ $\mathrm{Hz}), 3.25(1 \mathrm{H}$, br d, $J \sim 5 \mathrm{~Hz}), 3.23(1 \mathrm{H}$, br t, $J=7.7 \mathrm{~Hz}), 2.80(1 \mathrm{H}, \mathrm{s}$ br, OH), 2.05-2.00 (2H, m), 1.16 $(9 \mathrm{H}, \mathrm{s}) ;{ }^{13} \mathrm{C}$ NMR $\delta$ 138.2, 138.0, 137.7, 73.1 (C) 128.5 (x 2), 128.4 (x 5), 128.3 (x 2), 127.9 (x 2), 127.7 (x 3), 127.6, 78.0, 75.3, 75.1, 69.8, $68.2(\mathrm{CH}), 73.5,73.3,71.0,62.8,59.5,32.7\left(\mathrm{CH}_{2}\right), 27.5$ (x 3) $\left(\mathrm{CH}_{3}\right)$; IR $v_{\max } 3400$ (br, OH) cm ${ }^{-1}$; HR EIMS m/z (\%rel. int.) $553.3156\left(\mathrm{M}+\mathrm{H}^{+}, 1\right), 496$ (1), 339 (3), 299 (5), 181 (10), 163 (14), 91 (100). Calcd. for $\mathrm{C}_{33} \mathrm{H}_{45} \mathrm{O}_{7}, 553.3165$. Anal. Calcd. for $\mathrm{C}_{33} \mathrm{H}_{44} \mathrm{O}_{7}: \mathrm{C}_{\text {, }}$ 71.71; H, 8.02. Found: C, 71.57; H, 8.10.

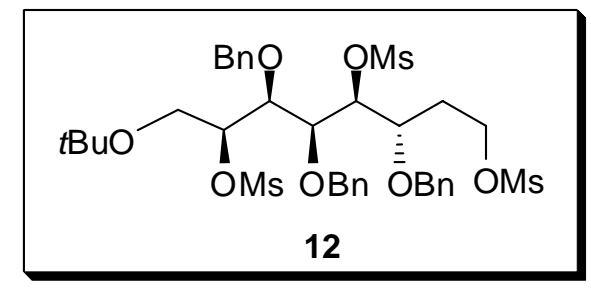

(3S,4S,5S,6S,7S)-3,5,6-Tris(benzyloxy)-8-tert-butoxyoctane-1,4,7-triyl tris(methanesulfonate) (12). A solution of triol $11(110 \mathrm{mg}$, ca. $0.2 \mathrm{mmol})$ in dry $\mathrm{CH}_{2} \mathrm{Cl}_{2}(2 \mathrm{~mL})$ was treated at $0{ }^{\circ} \mathrm{C}$ under $\mathrm{N}_{2}$ with $\mathrm{MsCl}(93 \mu \mathrm{L}, 1.2 \mathrm{mmol}), \mathrm{Et}_{3} \mathrm{~N}(250 \mu \mathrm{L}, 1.8 \mathrm{mmol})$ and DMAP $(1 \mathrm{mg}$, ca. $0.01 \mathrm{mmol})$. The reaction 
mixture was stirred for $2 \mathrm{~h}$ at the same temperature. Work-up (extraction with $\mathrm{CH}_{2} \mathrm{Cl}_{2}$ ) provided the crude trimesylate 12, which was directly used in the next step.

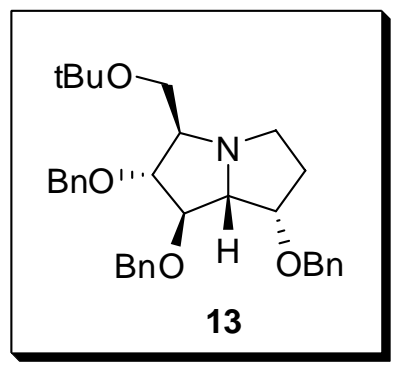

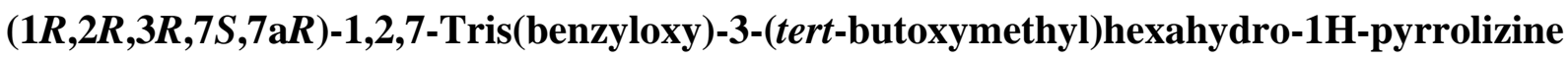

The crude trimesylate 12 from above was dissolved under $\mathrm{N}_{2}$ in dry DMSO $(3 \mathrm{~mL})$ and treated with $\mathrm{BnNH}_{2}(65 \mu \mathrm{L}, 0.6 \mathrm{mmol})$ and $\mathrm{NaI}(3 \mathrm{mg}, 0.02 \mathrm{mmol})$. The reaction mixture was then stirred at $80{ }^{\circ} \mathrm{C}$ for $24 \mathrm{~h}$. Work-up (extraction with EtOAc) and column chromatography on silica gel (first, hexanesEtOAc, 20:80, then EtOAc) furnished pyrrolizidine 13 (62 mg, 60\% overall from 11): colorless oil; $[\alpha]_{\mathrm{D}}+36.1\left(\mathrm{c} 1.2 ; \mathrm{CHCl}_{3}\right) ;{ }^{1} \mathrm{H} \mathrm{NMR} \delta 7.35-7.25(15 \mathrm{H}$, br m), $4.78(1 \mathrm{H}, J=11.8 \mathrm{~Hz}), 4.68(1 \mathrm{H}, \mathrm{d}, J=$ $11.8 \mathrm{~Hz}), 4.62(1 \mathrm{H}, \mathrm{d}, J=12 \mathrm{~Hz}), 4.55-4.50(2 \mathrm{H}, \mathrm{m}), 4.46(1 \mathrm{H}, \mathrm{d}, J=11.7 \mathrm{~Hz}), 4.40(1 \mathrm{H}, \mathrm{d}, J=12$ $\mathrm{Hz}), 4.10(1 \mathrm{H}$, br t, $J \sim 7.5 \mathrm{~Hz}), 3.90(1 \mathrm{H}, \mathrm{m}), 3.50(2 \mathrm{H}, \mathrm{m}), 3.35(1 \mathrm{H}, \mathrm{dd}, J=9,6.6 \mathrm{~Hz}), 3.17(1 \mathrm{H}$, br t, $J \sim 8 \mathrm{~Hz}), 2.90(1 \mathrm{H}, \mathrm{m}), 2.75(1 \mathrm{H}, \mathrm{td}, \mathrm{J}=10.5,6.2 \mathrm{~Hz}), 2.16(1 \mathrm{H}, \mathrm{br} \mathrm{dd}, J=13.2,6 \mathrm{~Hz}), 1.80(1 \mathrm{H}, \mathrm{m})$,

$1.19(9 \mathrm{H}, \mathrm{s}) ;{ }^{13} \mathrm{C} \mathrm{NMR} \delta 138.9,138.7,138.5,72.6$ (C), 127.4 (x 2), 128.3 (x 2), 128.2 (x 2), 127.7 (x 3), 127.6 (x 3), 127.4 (x 2), 127.3, 86.8, 81.4, 78.4, 71.3, $69.4(\mathrm{CH}), 72.4,72.2,70.7,64.1,52.5,32.0$ $\left(\mathrm{CH}_{2}\right), 27.5$ (x 3) $\left(\mathrm{CH}_{3}\right)$; HR EIMS m/z (\% rel. int.) $515.3014\left(\mathrm{M}^{+}, 1\right), 428$ (100), 246 (6), 91 (34). Calcd. for $\mathrm{C}_{33} \mathrm{H}_{41} \mathrm{NO}_{4}, 515.3035$.

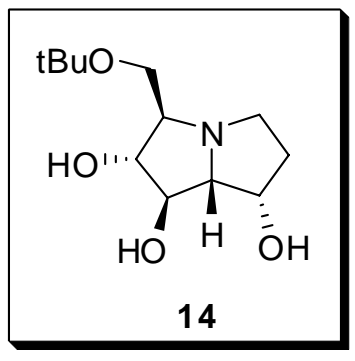

(1R,2R,3R,7S,7aR)-3-(Tert-butoxymethyl)hexahydro-1H-pyrrolizine-1,2,7-triol (14). 20\% $\mathrm{Pd}(\mathrm{OH})_{2}$ catalyst $(5 \mathrm{mg})$ was suspended in $\mathrm{EtOH}(1 \mathrm{~mL})$ and stirred under a $\mathrm{H}_{2}$ atmosphere. A solution of compound $13(51 \mathrm{mg}, 0.1 \mathrm{mmol})$ in $\mathrm{EtOH}(1 \mathrm{~mL})$ was then added via syringe to the catalyst suspension. The reaction mixture was stirred at room temperature for 2 days and then filtered through Celite. Solvent removal in vacuo gave crude $\mathbf{1 4}$ as an oily residue, which was used without purification in the next reaction. 


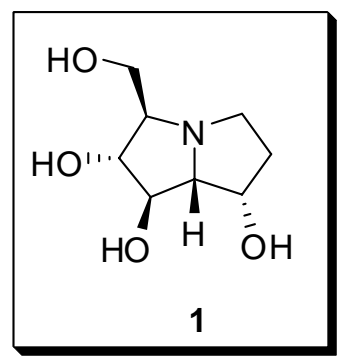

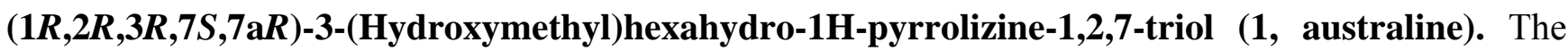
crude compound 14 from above was dissolved at $0{ }^{\circ} \mathrm{C}$ in a 1:1 $\mathrm{CH}_{2} \mathrm{Cl}_{2} / \mathrm{TFA}$ mixture $(1 \mathrm{~mL})$. The reaction mixture was then stirred at room temperature for $16 \mathrm{~h}$. After recooling to $0{ }^{\circ} \mathrm{C}$, aq $\mathrm{NH}_{3}(25 \%$ in water) was added dropwise until the reaction medium had a basic $\mathrm{pH}$. Removal of all volatiles under reduced pressure gave an oily residue which was purified by column chromatography on silica gel (first $\mathrm{CHCl}_{3}-\mathrm{MeOH}$ 1:1, then $\mathrm{CHCl}_{3}-\mathrm{MeOH}$-aq $\left.\mathrm{NH}_{3}, 5: 5: 1\right)$. This afforded pure australine 1 (15 mg, 78\% overall from 13): $[\alpha]_{D}+9.8\left(\right.$ c $\left.0.5 ; \mathrm{H}_{2} \mathrm{O}\right)$, lit. $^{3}$ (see next page) $[\alpha]_{\mathrm{D}}+8\left(\right.$ c $\left.0.35 ; \mathrm{H}_{2} \mathrm{O}\right)$; IR $v_{\max } 3340$ (br, $\mathrm{OH}) \mathrm{cm}^{-1}$; HR FABMS m/z $190.1071\left(\mathrm{M}+\mathrm{H}^{+}\right)$. Calcd. for $\mathrm{C}_{8} \mathrm{H}_{16} \mathrm{NO}_{4}, 190.1079$.

NMR chemical shifts of australine are very sensitive to the $\mathrm{pH}$ of the medium. This reflects the coexistence in solution of free and protonated base. The following tables show the data reported hitherto in the literature for natural and synthetic australine. Our data have been measured in $\mathrm{D}_{2} \mathrm{O}$ which had been added a few drops of $\mathrm{NaOD}$ solution in $\mathrm{D}_{2} \mathrm{O}$ in order to make the medium basic (in pure $\mathrm{D}_{2} \mathrm{O}$, the spectra turned out to be essentially those of protonated australine). Sodium 3(trimethylsilyl)propane- $\mathrm{d}_{6}$-sulfonate was added as the internal reference $(\delta=0)$ for ${ }^{1} \mathrm{H}$ and ${ }^{13} \mathrm{C}$ chemical shift values. Comparison was carried out with an authentic sample of the natural compound (NMR spectra of the mixture were measured). 


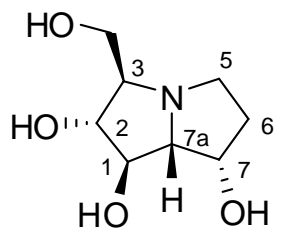

Table. Comparison of published ${ }^{1} \mathrm{H}$ NMR data of australine.

\begin{tabular}{|c|c|c|c|c|c|c|}
\hline Atom & $\begin{array}{l}\text { Wormald et al. } \\
\text { (in } \mathrm{D}_{2} \mathrm{O}, \mathrm{pH} \\
\text { adjusted to } 8.6 \text { ) }\end{array}$ & $\begin{array}{l}\text { Denmark et al. } \\
\quad\left(\text { in } \mathrm{D}_{2} \mathrm{O}\right)\end{array}$ & $\begin{array}{l}\text { Pearson et al. } \\
\quad\left(\text { in } \mathrm{D}_{2} \mathrm{O}\right)\end{array}$ & $\begin{array}{l}\text { Wong et al. } \\
\quad\left(\text { in } \mathrm{D}_{2} \mathrm{O}\right)\end{array}$ & $\begin{array}{l}\text { White et al. } \\
\quad\left(\text { in } \mathrm{D}_{2} \mathrm{O}\right)\end{array}$ & $\begin{array}{c}\text { This paper } \\
\left.\text { (in } \mathrm{D}_{2} \mathrm{O}+\mathrm{NaOD}\right)\end{array}$ \\
\hline H-1 & $\begin{array}{l}\text { 4.291 (dd, } J= \\
8.2,7.4 \mathrm{~Hz})\end{array}$ & $\begin{array}{l}\mathbf{4 . 0 4}(\mathrm{t}, J=7.8 \\
\mathrm{Hz})\end{array}$ & $\begin{array}{l}\mathbf{4 . 0 8}(\mathrm{t}, J=7.5 \\
\mathrm{Hz})\end{array}$ & $\begin{array}{l}\mathbf{4 . 1 4}(\mathrm{t}, J=7.9 \\
\mathrm{Hz})\end{array}$ & $\begin{array}{l}4.25(\mathrm{t}, J=8 \\
\mathrm{Hz})\end{array}$ & $\begin{array}{l}\mathbf{4 . 0 9}(\mathrm{t}, J=6.8 \\
\mathrm{Hz})\end{array}$ \\
\hline H-2 & $\begin{array}{l}3.959(\mathrm{dd}, J= \\
9.5,8.2 \mathrm{~Hz})\end{array}$ & $\begin{array}{l}3.71(\mathrm{dd}, J= \\
9.5,8.3 \mathrm{~Hz})\end{array}$ & $\begin{array}{l}3.73(\mathrm{t}, J=8.5 \\
\mathrm{Hz})\end{array}$ & $\begin{array}{l}3.80(\mathrm{dd}, J= \\
9.4,8.1 \mathrm{~Hz})\end{array}$ & $\begin{array}{l}3.91(\mathrm{t}, J=9 \\
\mathrm{Hz})\end{array}$ & $\begin{array}{l}3.79(\mathrm{t}, J=7.5 \\
\mathrm{Hz})\end{array}$ \\
\hline H-3 & $2.803(\mathrm{~m})$ & $\begin{array}{l}\text { 2.58-2.52 } \\
(\mathrm{m}, 2 \mathrm{H})\end{array}$ & $\begin{array}{l}\mathbf{2 . 7 0 - 2 . 5 0} \\
(\mathrm{m}, 2 \mathrm{H})\end{array}$ & $\begin{array}{l}\mathbf{2 . 6 4 - 2 . 6 1} \\
(\mathrm{m}, 2 \mathrm{H})\end{array}$ & $\begin{array}{l}\text { 2.78-2.72 } \\
(\mathrm{m}, 2 \mathrm{H})\end{array}$ & $\begin{array}{l}\begin{array}{l}\text { 2.75-2.65 } \\
(\mathrm{m}, 2 \mathrm{H})\end{array} \\
\end{array}$ \\
\hline H-5 & $\begin{array}{l}\mathbf{2 . 8 0 4}(\mathrm{ddd}, J= \\
*, 11.5,6 \mathrm{~Hz})\end{array}$ & $\begin{array}{l}2.58-2.52 \\
(\mathrm{~m}, 2 \mathrm{H}) \\
\end{array}$ & $\begin{array}{l}\mathbf{2 . 7 0 - 2 . 5 0} \\
(\mathrm{m}, 2 \mathrm{H})\end{array}$ & $\begin{array}{l}\mathbf{2 . 6 4 - 2 . 6 1} \\
(\mathrm{m}, 2 \mathrm{H}) \\
\end{array}$ & $\begin{array}{l}\mathbf{2 . 7 8 - 2 . 7 2} \\
(\mathrm{m}, 2 \mathrm{H}) \\
\end{array}$ & $\begin{array}{l}2.75-2.65 \\
(\mathrm{~m}, 2 \mathrm{H}) \\
\end{array}$ \\
\hline H-5' & $\begin{array}{l}3.229(\mathrm{ddd}, J= \\
*, 7.5,2.1 \mathrm{~Hz})\end{array}$ & $\begin{array}{l}2.98(\mathrm{ddd}, J= \\
9.8,7.6,2.2 \mathrm{~Hz})\end{array}$ & $\begin{array}{l}\text { 3.10-2.90 } \\
(\mathrm{m}, 2 \mathrm{H})\end{array}$ & $\begin{array}{l}\text { 3.05 }(\mathrm{ddd}, J= \\
9.9,7.5,2.2 \mathrm{~Hz})\end{array}$ & $\begin{array}{l}3.22-3.15 \\
(\mathrm{~m}, 2 \mathrm{H})\end{array}$ & $\begin{array}{l}3.12 \\
(\mathrm{~m}, 1 \mathrm{H})\end{array}$ \\
\hline H-6 & $\begin{array}{l}2.097 \text { (dddd, } J= \\
*, 6,2.4,2.1 \mathrm{~Hz})\end{array}$ & $\begin{array}{l}\mathbf{1 . 8 7 - 1 . 8 2} \\
(\mathrm{m}, 1 \mathrm{H})\end{array}$ & $\begin{array}{l}\text { 1.90-1.70 } \\
(\mathrm{m}, 2 \mathrm{H})\end{array}$ & $\begin{array}{l}\mathbf{1 . 9 5 - 1 . 9 2} \\
(\mathrm{m}, 1 \mathrm{H})\end{array}$ & $\begin{array}{l}\mathbf{2 . 0 6 - 2 . 0 2} \\
(\mathrm{m}, 1 \mathrm{H})\end{array}$ & $\begin{array}{l}\mathbf{1 . 9 5 - 1 . 9 0} \\
(\mathrm{m}, 1 \mathrm{H})\end{array}$ \\
\hline H-6' & $\begin{array}{l}2.002(\text { dddd, } J= \\
*, 11.5,7.5,4.2 \mathrm{~Hz})\end{array}$ & $\begin{array}{l}\text { 1.79-1.71 } \\
(\mathrm{m}, 1 \mathrm{H})\end{array}$ & $\begin{array}{l}\mathbf{1 . 9 0 - 1 . 7 0} \\
(\mathrm{m}, 2 \mathrm{H})\end{array}$ & $\begin{array}{l}\mathbf{1 . 8 7 - 1 . 8 2} \\
(\mathrm{m}, 1 \mathrm{H})\end{array}$ & $\begin{array}{l}\text { 2.01-1.92 } \\
(\mathrm{m}, 1 \mathrm{H})\end{array}$ & $\begin{array}{l}\mathbf{1 . 9 0 - 1 . 8 0} \\
(\mathrm{m}, 1 \mathrm{H})\end{array}$ \\
\hline H-7 & $\begin{array}{l}\text { 4.434 (ddd, } J= \\
4.4,4.2,2.4 \mathrm{~Hz})\end{array}$ & $\begin{array}{l}\mathbf{4 . 1 9}(\mathrm{dt}, \quad J= \\
2.2,4.2 \mathrm{~Hz})\end{array}$ & $4.21(\mathrm{~m})$ & $\begin{array}{l}4.27(\mathrm{dt}, \quad J= \\
2.2,4 \mathrm{~Hz})\end{array}$ & $4.39(\mathrm{~s})$ & $4.29(\mathrm{~m})$ \\
\hline $\mathrm{H}-7 \mathrm{a}$ & $\begin{array}{l}3.272(\mathrm{dd}, J= \\
7.4,4.4 \mathrm{~Hz})\end{array}$ & $\begin{array}{l}3.02(\mathrm{dd}, \quad J= \\
7.6,4.4 \mathrm{~Hz})\end{array}$ & $\begin{array}{l}\mathbf{3 . 1 0 - 2 . 9 0} \\
(\mathrm{m}, 2 \mathrm{H})\end{array}$ & $\begin{array}{l}\text { 3.09 }(\mathrm{dd}, J= \\
7.5,4.3 \mathrm{~Hz})\end{array}$ & $\begin{array}{l}\text { 3.22-3.15 } \\
(\mathrm{m}, 2 \mathrm{H})\end{array}$ & $\begin{array}{l}3.15(\mathrm{dd}, \quad J= \\
7.5,4.5 \mathrm{~Hz}, 1 \mathrm{H})\end{array}$ \\
\hline \multirow[t]{2}{*}{$\mathrm{CH}_{2} \mathrm{OH}$} & $3.850(\mathrm{~m})$ & $\begin{array}{l}3.60(\mathrm{dd}, \quad J= \\
12,3.7 \mathrm{~Hz})\end{array}$ & $\begin{array}{l}3.63(\mathrm{dd}, J= \\
11,3 \mathrm{~Hz})\end{array}$ & $\begin{array}{l}3.70(\mathrm{dd}, J= \\
11.8,3.5 \mathrm{~Hz})\end{array}$ & $\begin{array}{l}3.81(\mathrm{dd}, J= \\
12,3 \mathrm{~Hz})\end{array}$ & $\begin{array}{l}3.75(\mathrm{dd}, \quad J= \\
11.3,3.8 \mathrm{~Hz})\end{array}$ \\
\hline & $3.677(\mathrm{~m})$ & $\begin{array}{l}3.43(\mathrm{dd}, J= \\
12,6.6 \mathrm{~Hz})\end{array}$ & $\begin{array}{l}3.45(\mathrm{t}, J=11, \\
6.5 \mathrm{~Hz})\end{array}$ & $\begin{array}{l}3.51(\mathrm{dd}, J= \\
11.8,6.6 \mathrm{~Hz})\end{array}$ & $\begin{array}{l}3.73(\mathrm{dd}, J= \\
12,6 \mathrm{~Hz})\end{array}$ & $\begin{array}{l}3.57(\mathrm{dd}, J= \\
11.5,6.8 \mathrm{~Hz})\end{array}$ \\
\hline
\end{tabular}

${ }^{1}$ Wormald, M. R.; Nash, R. J.; Hrnciar, P.; White, J. D.; Molyneux, R. J.; Fleet, G. W. J. Tetrahedron:

Asymmetry 1998, 9, 2549-2558. *The geminal coupling constants of protons at C-5 and C-6 are not given.

Sodium 3-(trimethylsilyl)propanesulfonate was used as the internal reference $(\delta=0)$.

${ }^{2}$ Denmark, S. E.; Martinborough, E. A. J. Am. Chem. Soc. 1999, 121, 3046-3056. The solvent signal at $\delta 4.63$ was used as the internal reference.

${ }^{3}$ Pearson, W. H.; Hines, J. V. J. Org. Chem. 2000, 65, 5785-5793 (internal reference not specified).

${ }^{4}$ Romero, A.; Wong, C.-H. J. Org. Chem. 2000, 65, 8264-8268 (internal reference not specified).

${ }^{5}$ White, J. D.; Hrnciar, P. J. Org. Chem. 2000, 65, 9129-9142 (internal reference not specified, solvent for ${ }^{1} \mathrm{H}$ NMR was erroneously indicated to be $\mathrm{CDCl}_{3}$ in the main text).

${ }^{6}$ Chemical shift values are referenced to sodium 3-(trimethylsilyl)propanesulfonate- $\mathrm{d}_{6}(\delta=0)$. 


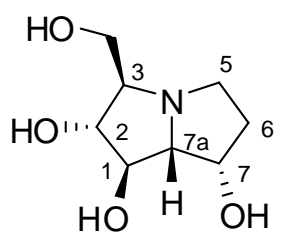

Table. Comparison of published ${ }^{13} \mathrm{C}$ NMR data of australine.

\begin{tabular}{|c|c|c|c|c|c|c|}
\hline Atom & $\begin{array}{l}\text { Kato et al. }{ }^{1} \\
\quad \text { (in } \mathrm{D}_{2} \mathrm{O} \text { ) }\end{array}$ & $\begin{array}{l}\text { Denmark et al. } \\
\quad\left(\text { in } \mathrm{D}_{2} \mathrm{O}\right)\end{array}$ & $\begin{array}{l}\text { Pearson et al. } \\
\quad\left(\text { in } \mathrm{D}_{2} \mathrm{O}\right)\end{array}$ & $\begin{array}{l}\text { Wong et al. } \\
\quad \text { (in } \mathrm{D}_{2} \mathrm{O} \text { ) }\end{array}$ & $\begin{array}{l}\text { White et al. } \\
\left.\quad \text { (in } \mathrm{D}_{2} \mathrm{O}\right)\end{array}$ & $\begin{array}{c}\text { This paper }^{6} \\
\text { (in } \mathrm{D}_{2} \mathrm{O}+\mathrm{NaOD} \text { ) }\end{array}$ \\
\hline C-1 & 75.9 & 72.69 & 73.9 & 72.2 & 73.4 & 77.2 \\
\hline C-2 & 73.5 & 78.43 & 71.7 & 69.8 & 71.0 & 75.3 \\
\hline C-3 & 73.3 & 70.22 & 71.4 & 69.7 & 70.8 & 75.3 \\
\hline C-5 & 54.6 & 51.58 & 52.7 & 51.0 & 52.1 & 55.2 \\
\hline C-6 & 38.0 & 34.90 & 35.9 & 34.4 & 35.4 & 38.2 \\
\hline C-7 & 81.8 & 69.09 & 79.5 & 78.0 & 79.1 & 82.8 \\
\hline$C-7 a$ & 72.3 & 70.47 & 70.3 & 68.6 & 69.8 & 72.5 \\
\hline $\mathrm{CH}_{2} \mathrm{OH}$ & 65.5 & 62.19 & 63.1 & 61.9 & 62.9 & 66.5 \\
\hline
\end{tabular}

${ }^{1}$ Kato, A.; Kano, E.; Adachi, I.; Molyneux, R. J.; Watson, A. A.; Nash, R. J.; Fleet, G. W. J.; Wormald, M. R.; Kizu, H.; Ikeda, K.; Asano, N. Tetrahedron: Asymmetry 2003, 14, 325-331. Sodium 3-(trimethylsilyl)propionate was used as internal reference. Signals were assigned with the aid of 2D NMR experiments.

${ }^{2}$ Denmark, S. E.; Martinborough, E. A. J. Am. Chem. Soc. 1999, 121, 3046-3056 (internal reference not specified). Some signal assignments do not coincide with those of Kato et al.

${ }^{3}$ Pearson, W. H.; Hines, J. V. J. Org. Chem. 2000, 65, 5785-5793 (dioxane as internal reference, $\delta 67.4$ ppm). Signals were not individually assigned and are arranged here according to Kato et al. ${ }^{1}$

${ }^{4}$ Romero, A.; Wong, C.-H. J. Org. Chem. 2000, 65, 8264-8268 (internal reference not specified). Signals were not individually assigned and are arranged here according to Kato et al. ${ }^{1}$

${ }^{5}$ White, J. D.; Hrnciar, P. J. Org. Chem. 2000, 65, 9129-9142 (residual methanol peak as internal reference, $\delta$ $49.15 \mathrm{ppm})$. Signals were not individually assigned and are arranged here according to Kato et al. ${ }^{1}$

${ }^{6}$ Chemical shift values are referenced to sodium 3-(trimethylsilyl)propanesulfonate- $\mathrm{d}_{6}(\delta=0)$. Signals have not been individually assigned and are arranged here according to Kato et al. ${ }^{1}$ 


\section{NMR Spectra of selected compounds}
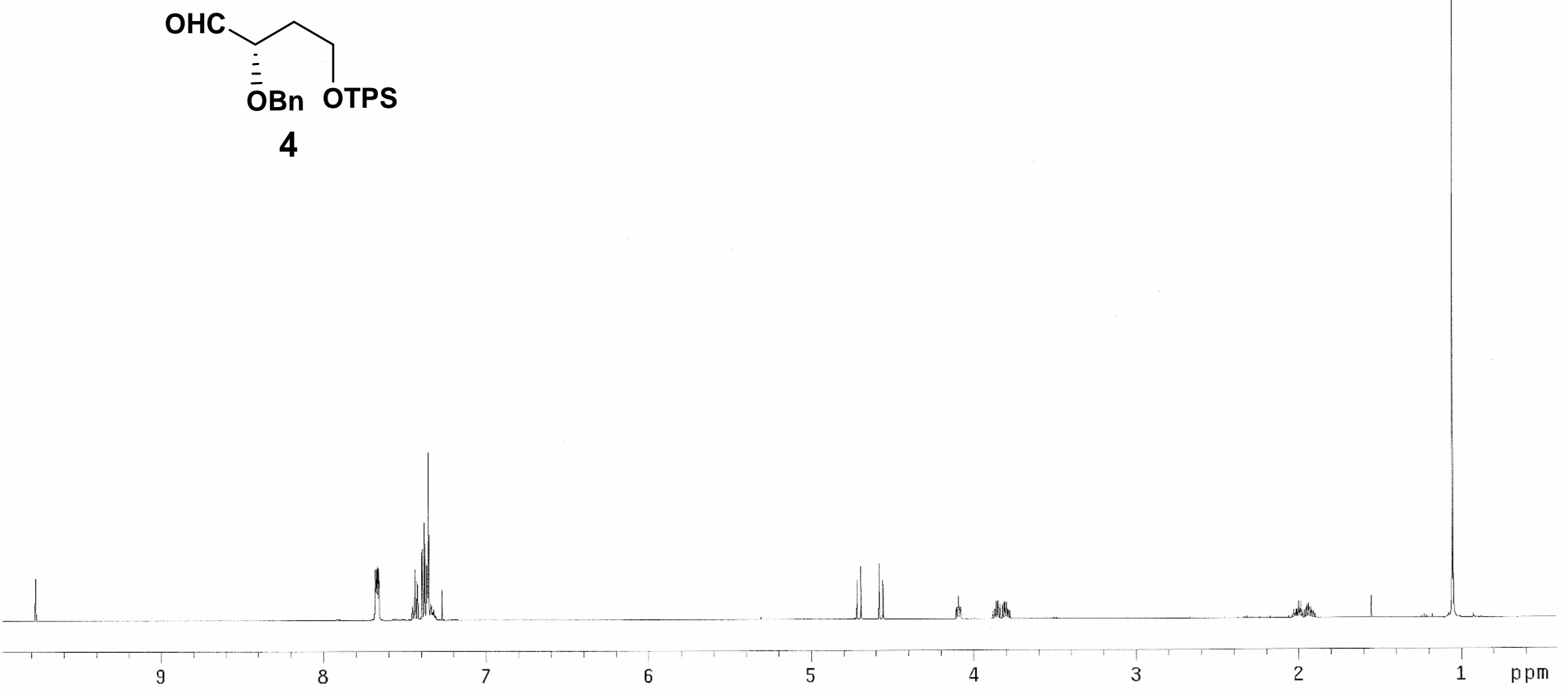


\section{$\mathrm{OHC}$ \\ ÖBn OTPS \\ 4}

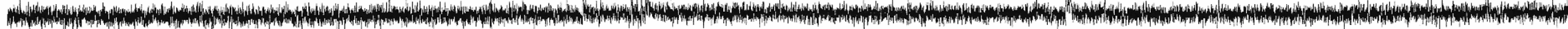

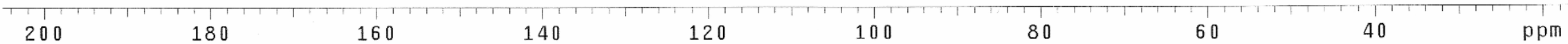



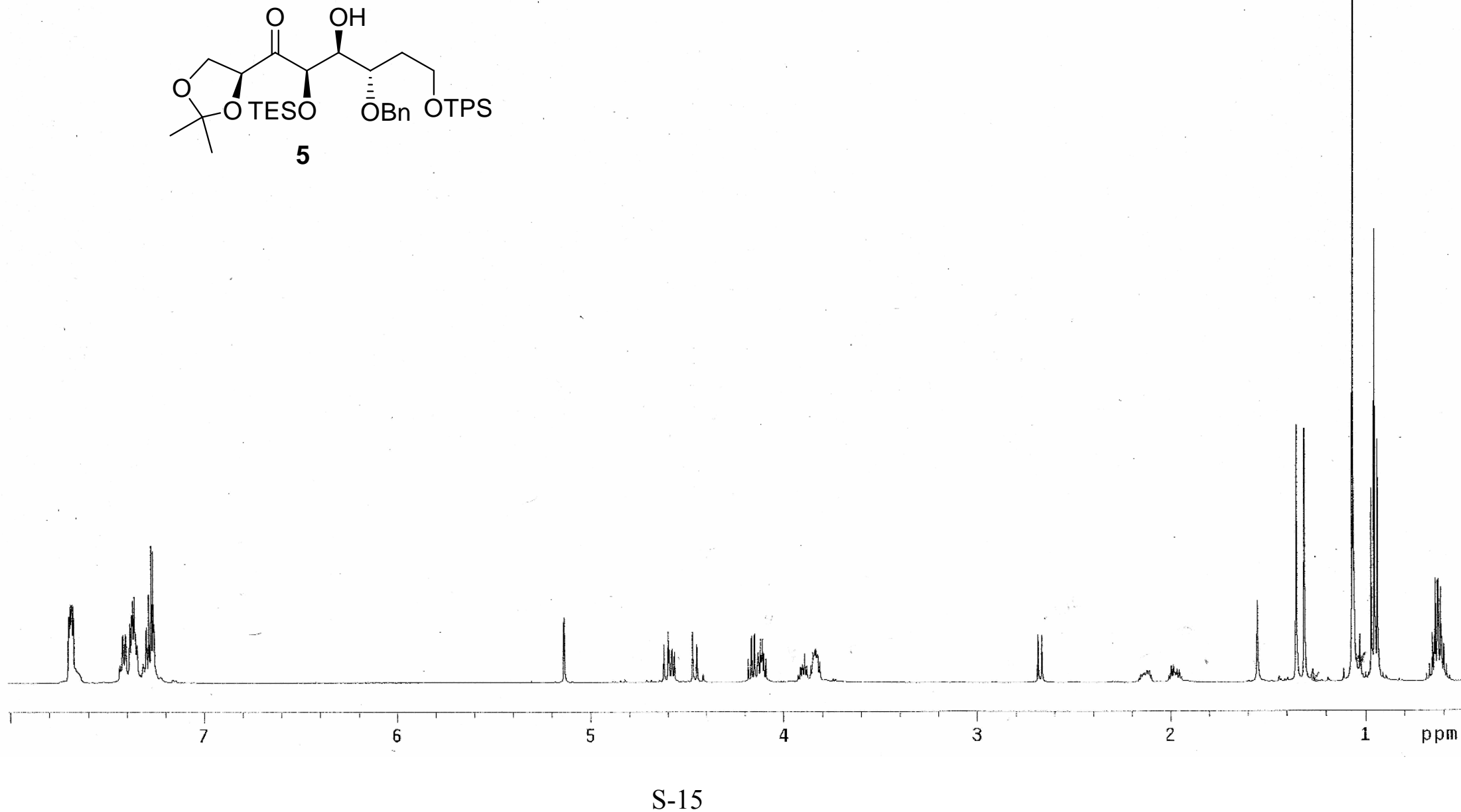

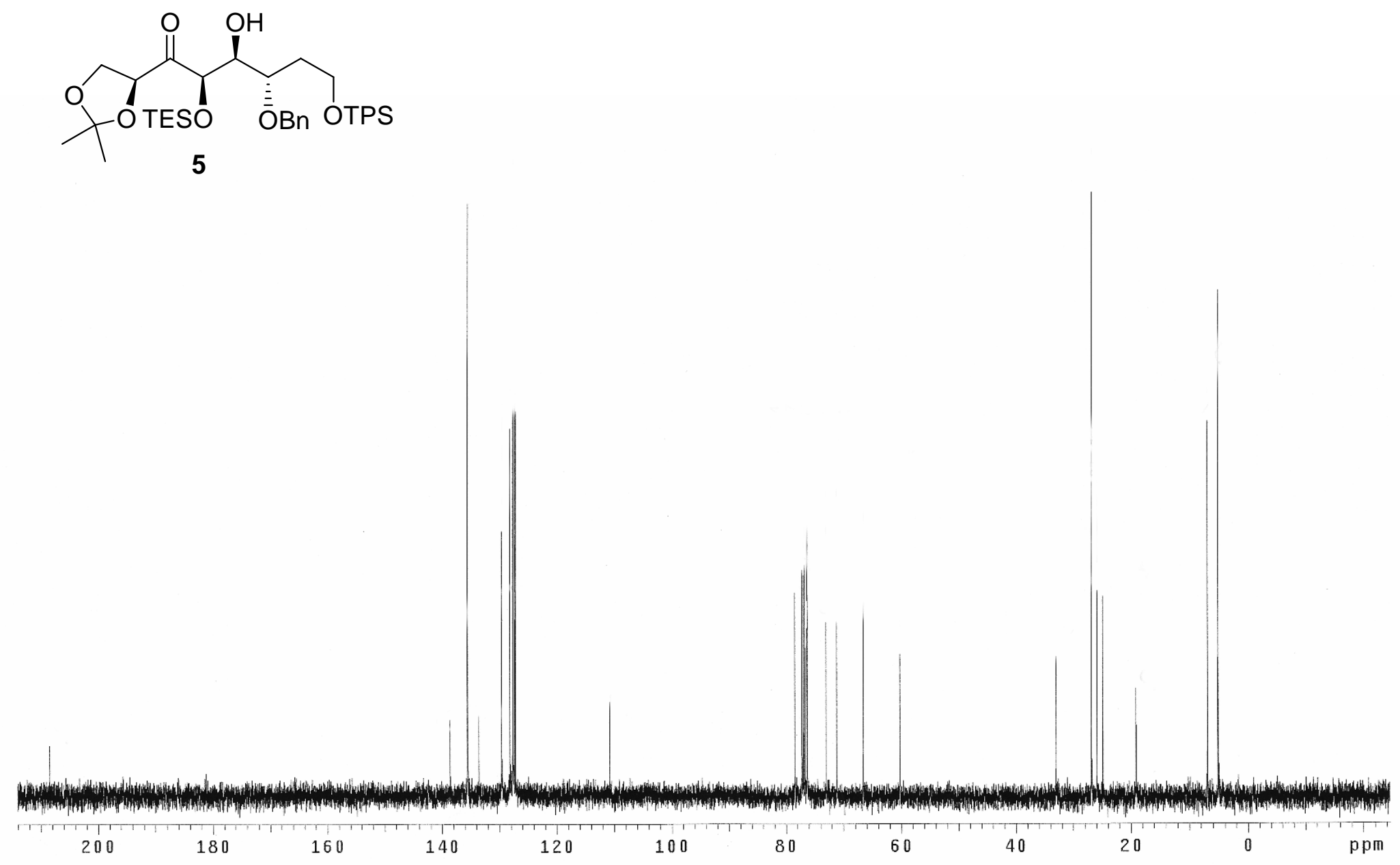

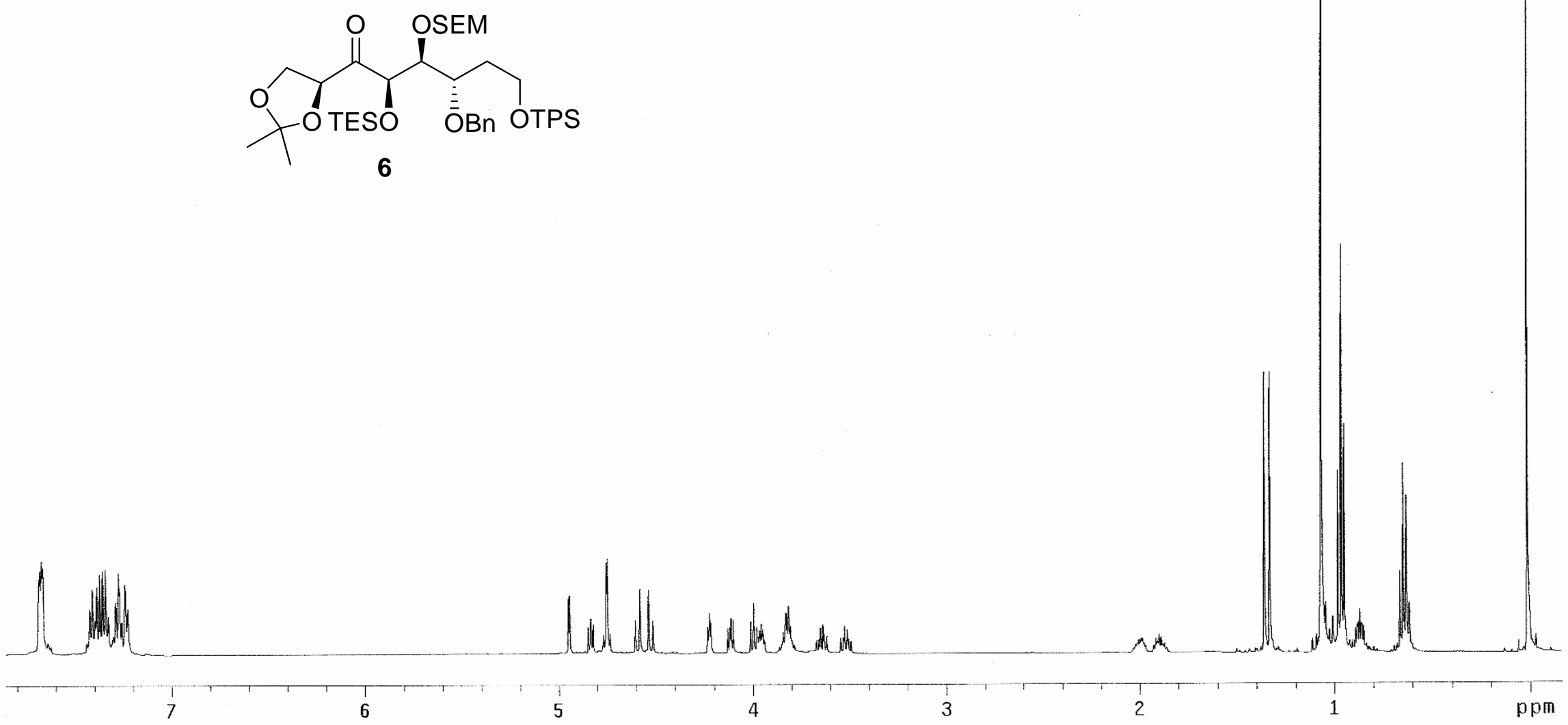

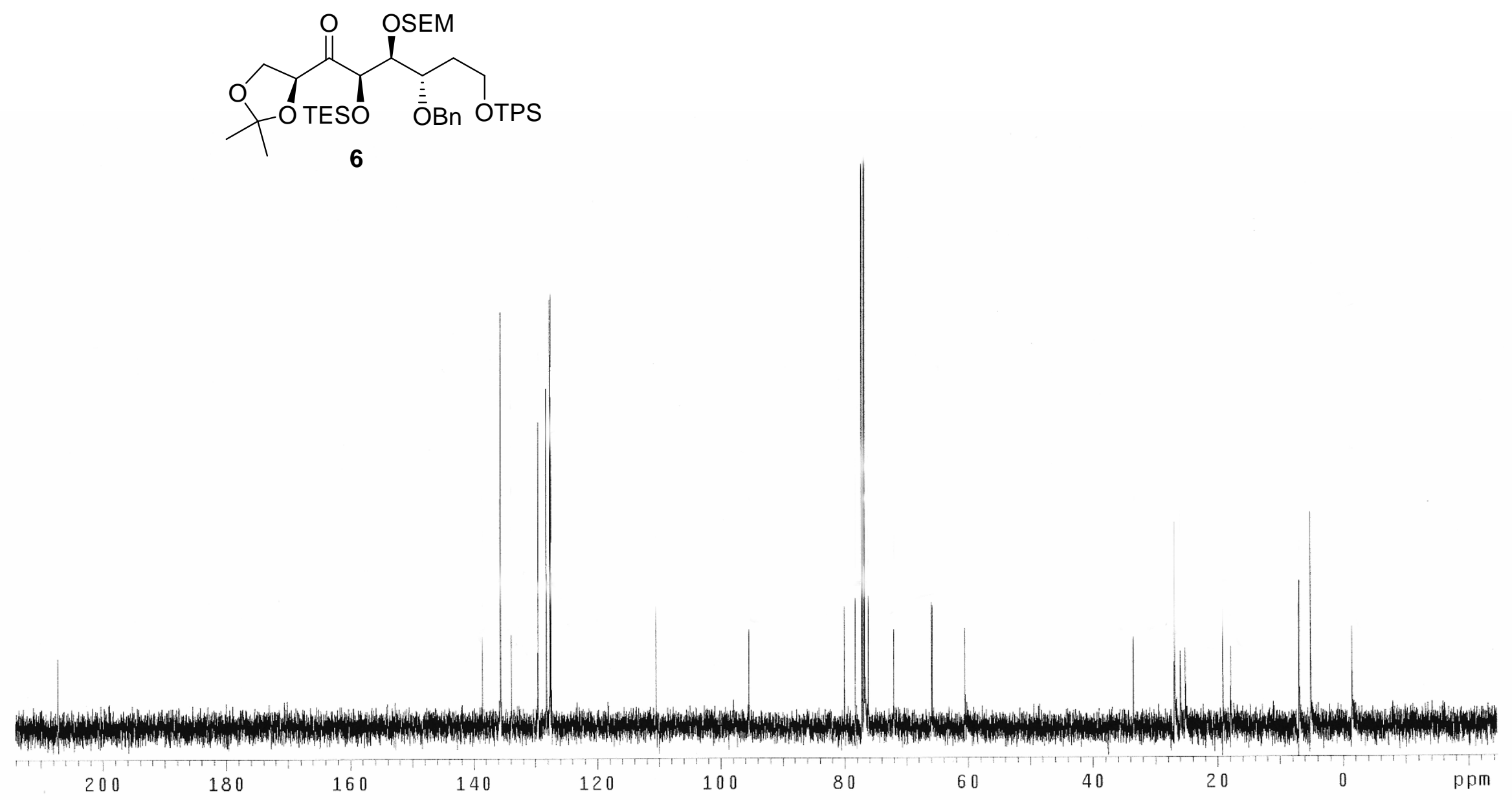

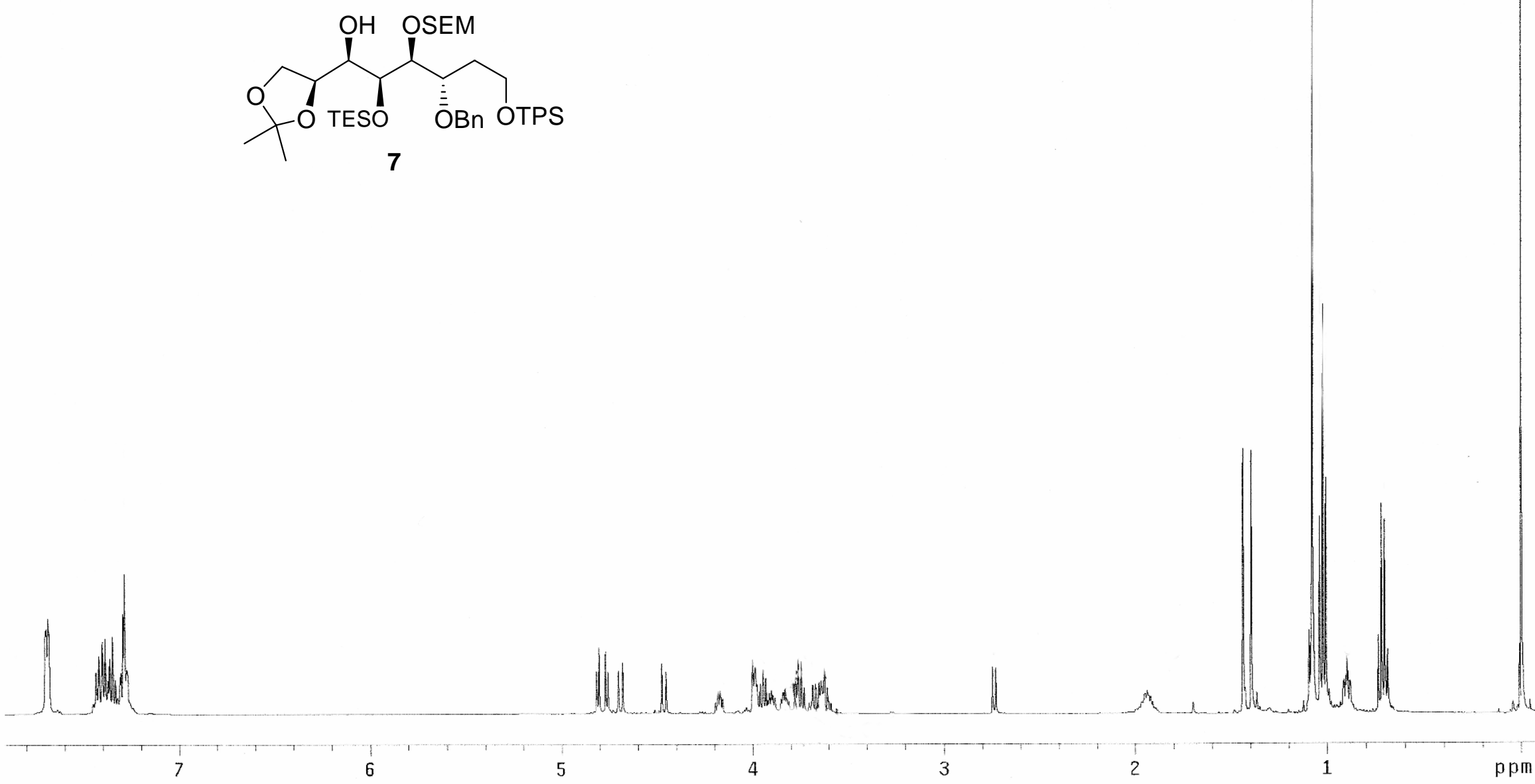

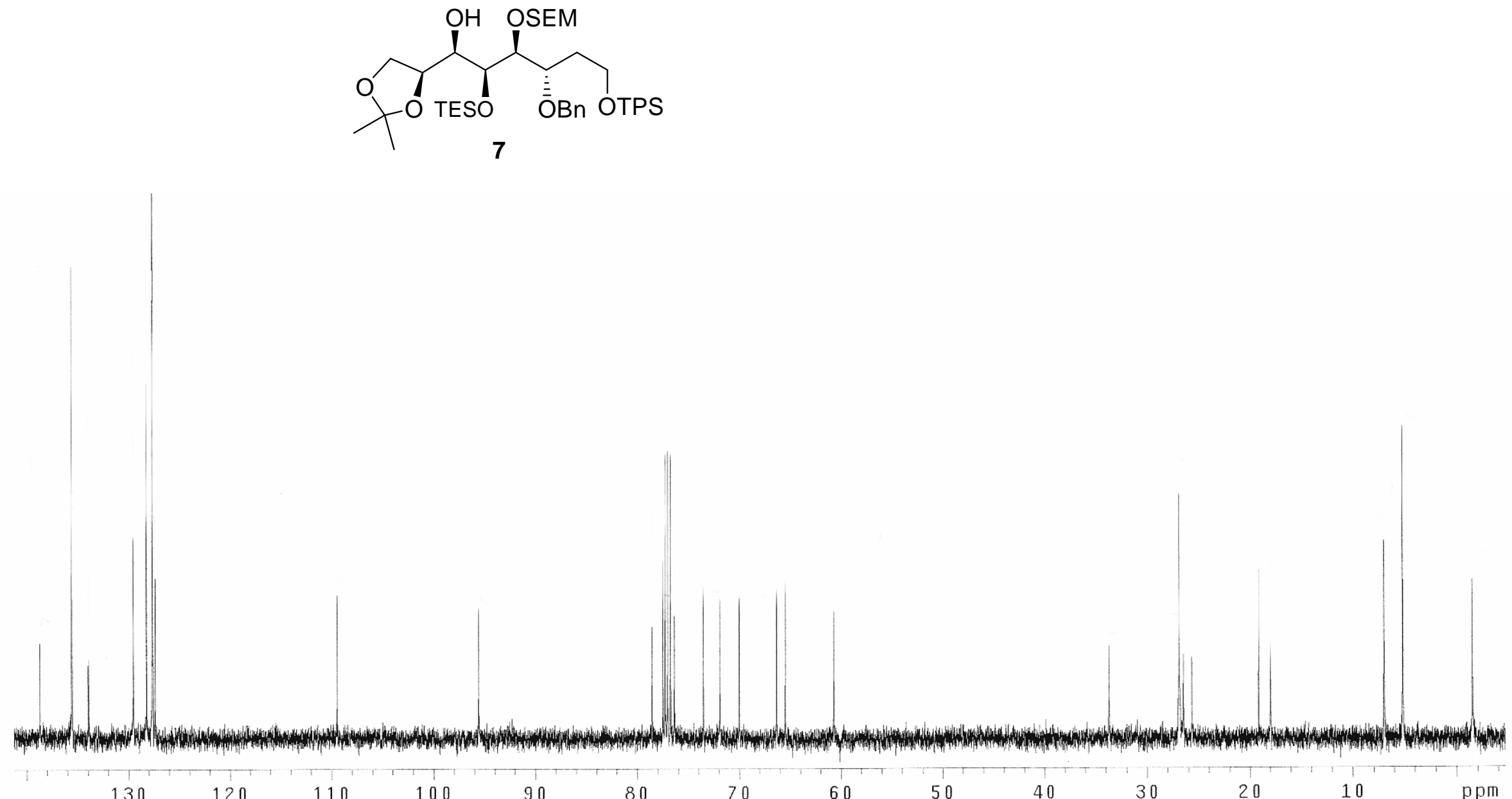

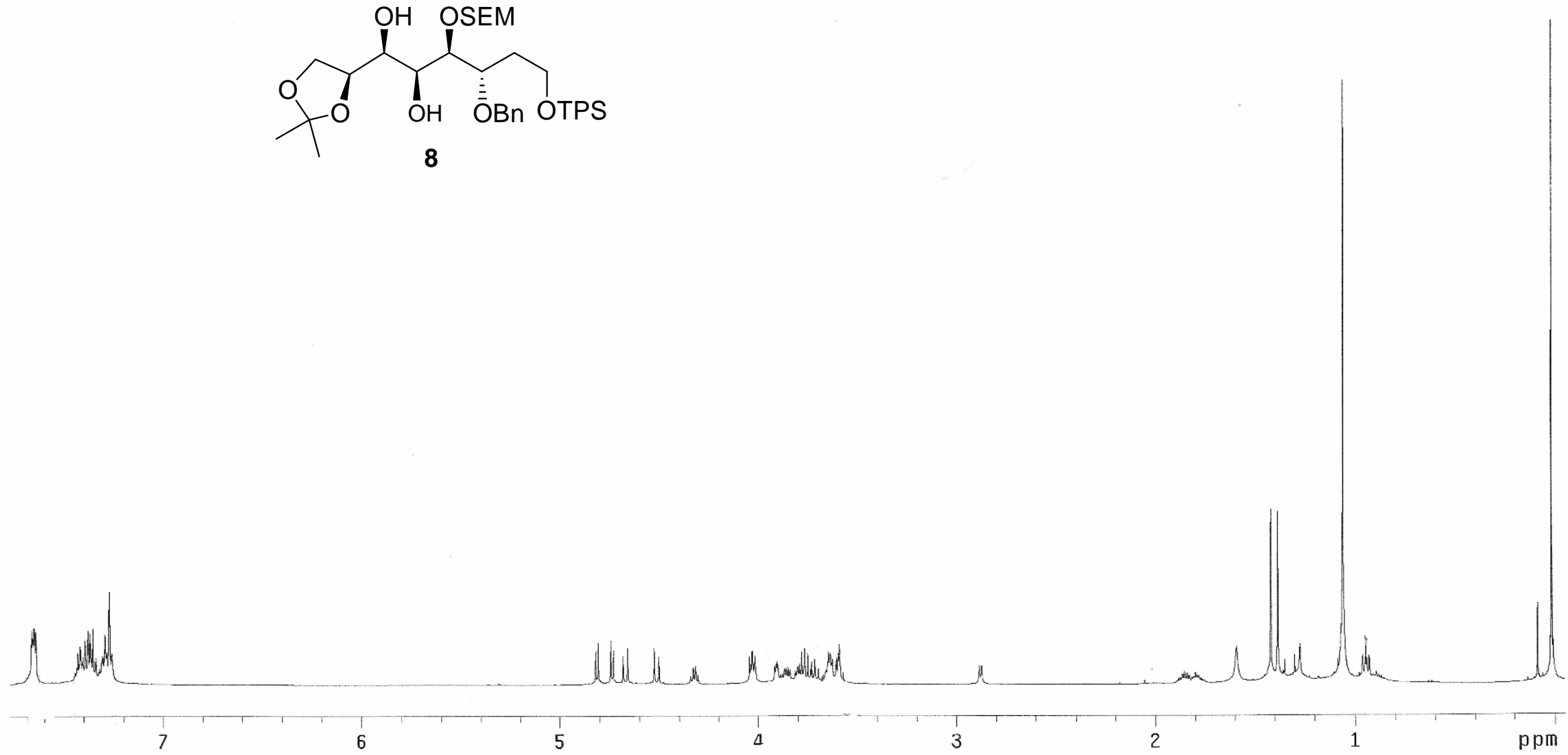


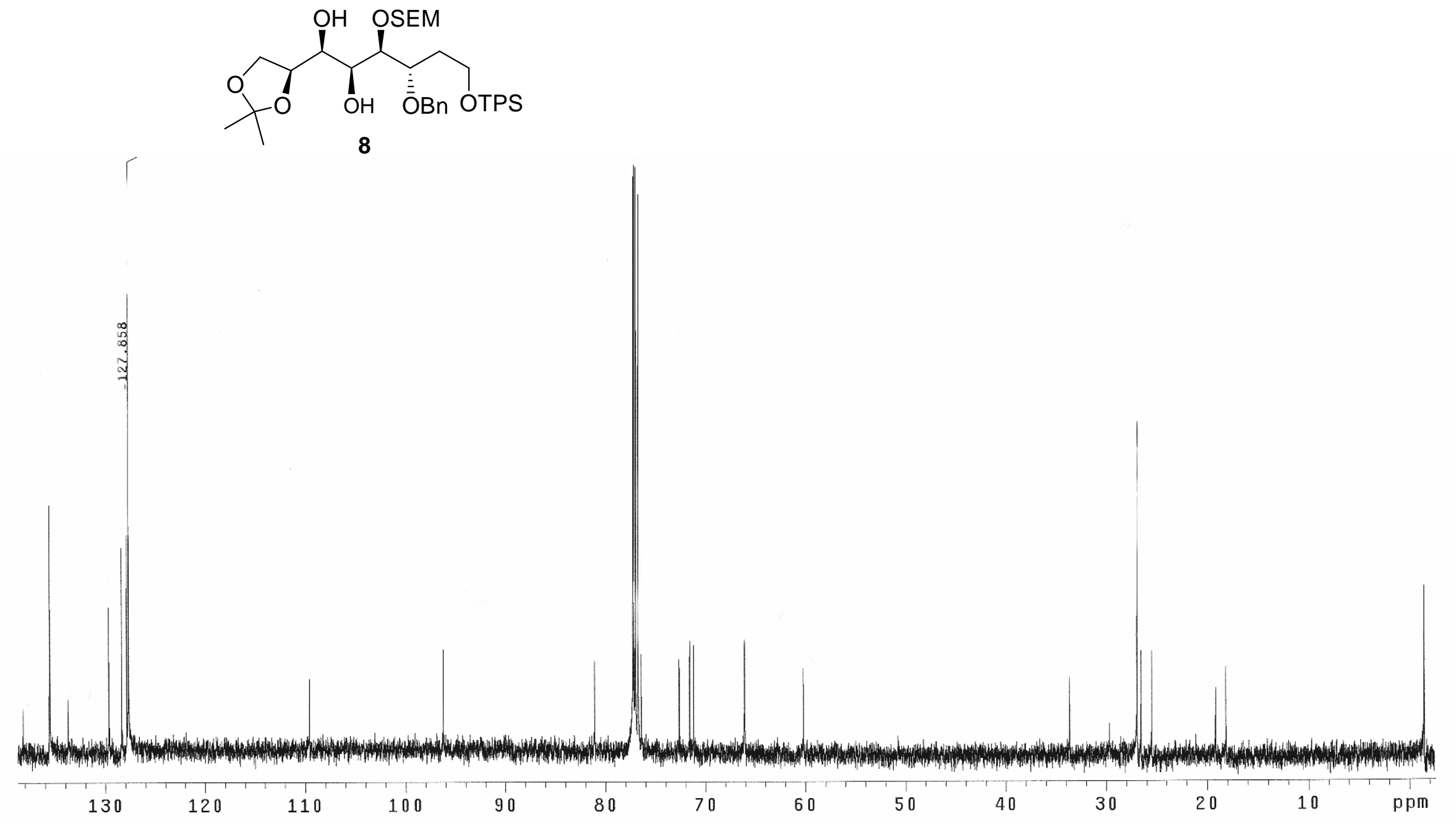



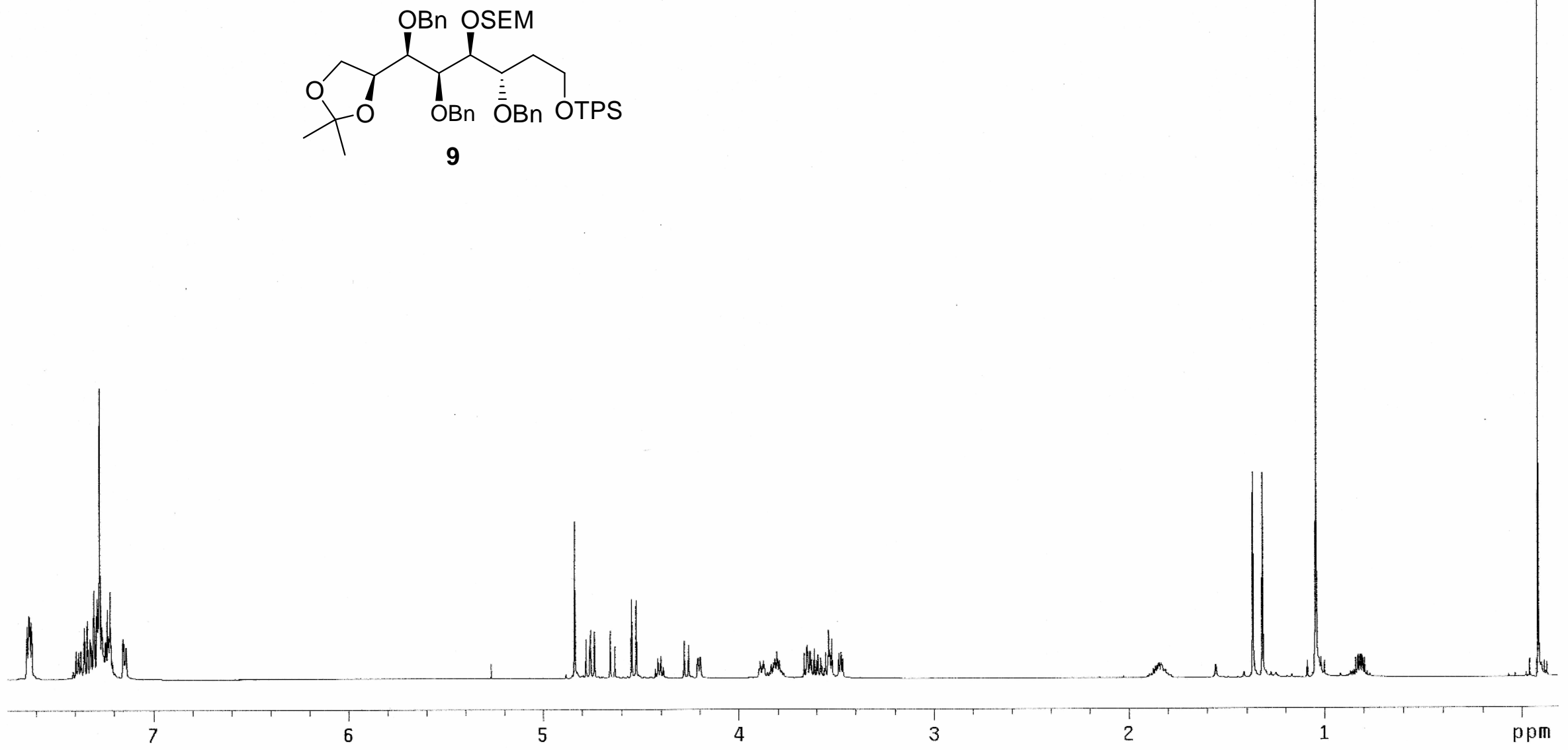

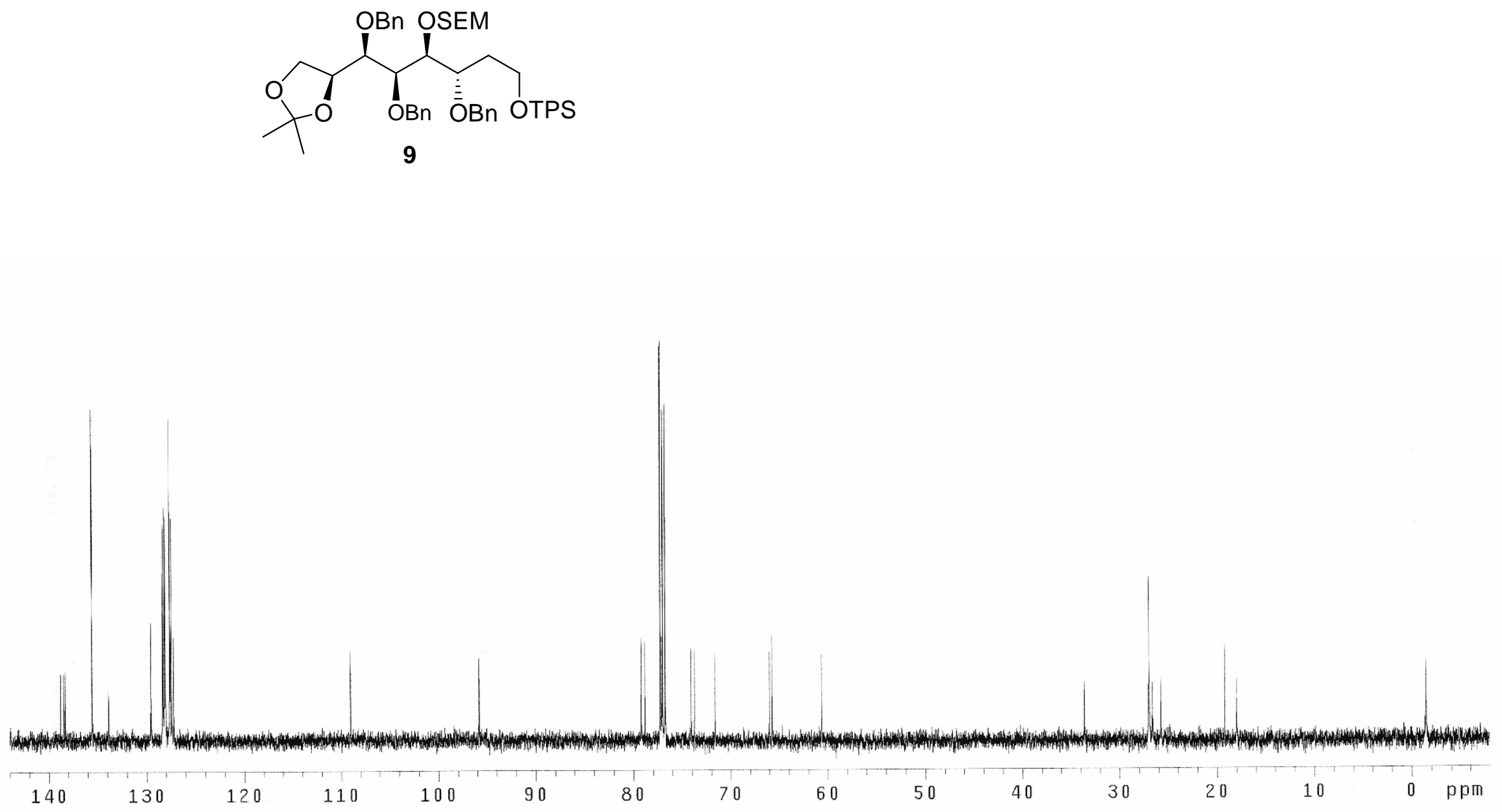

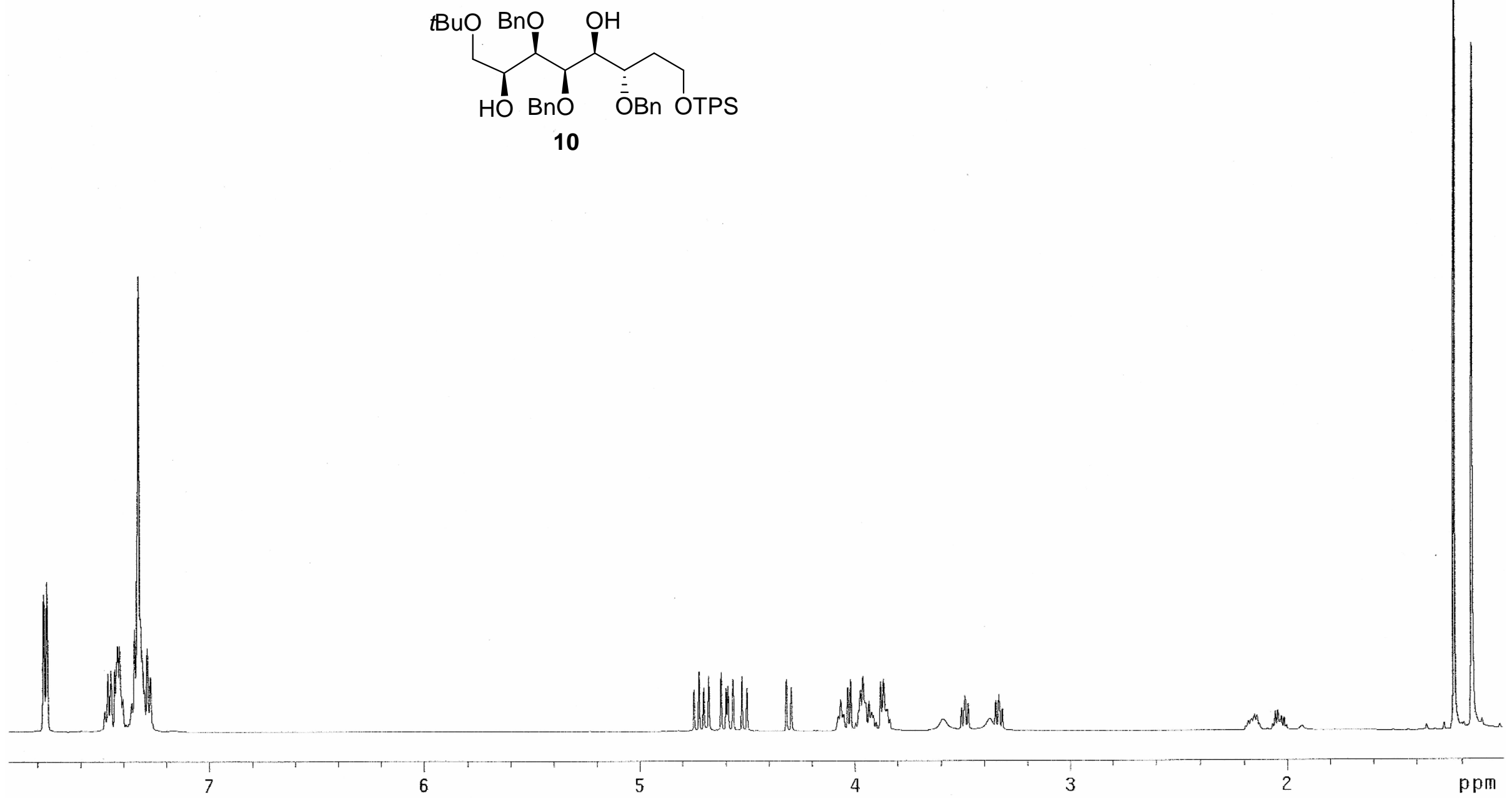


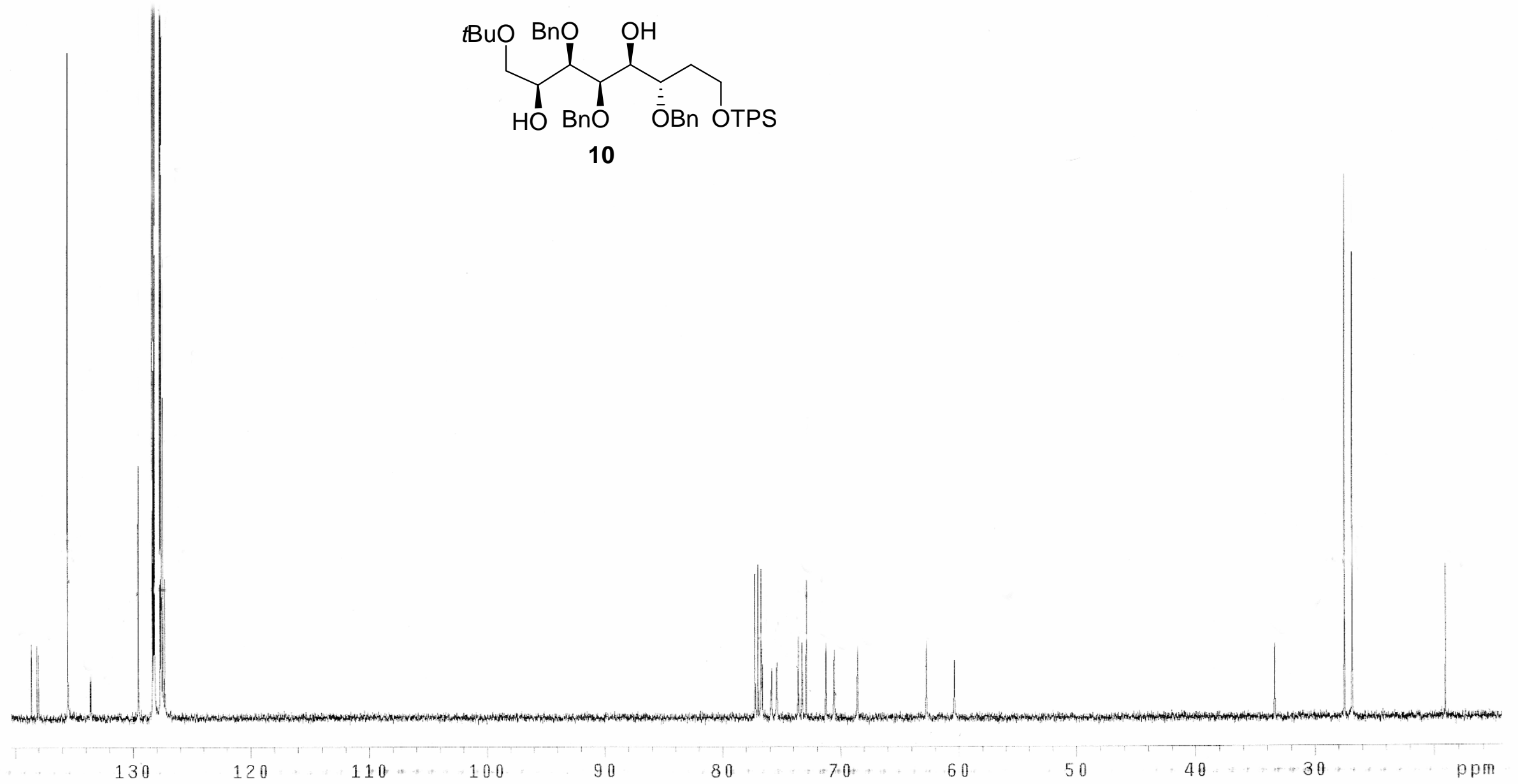



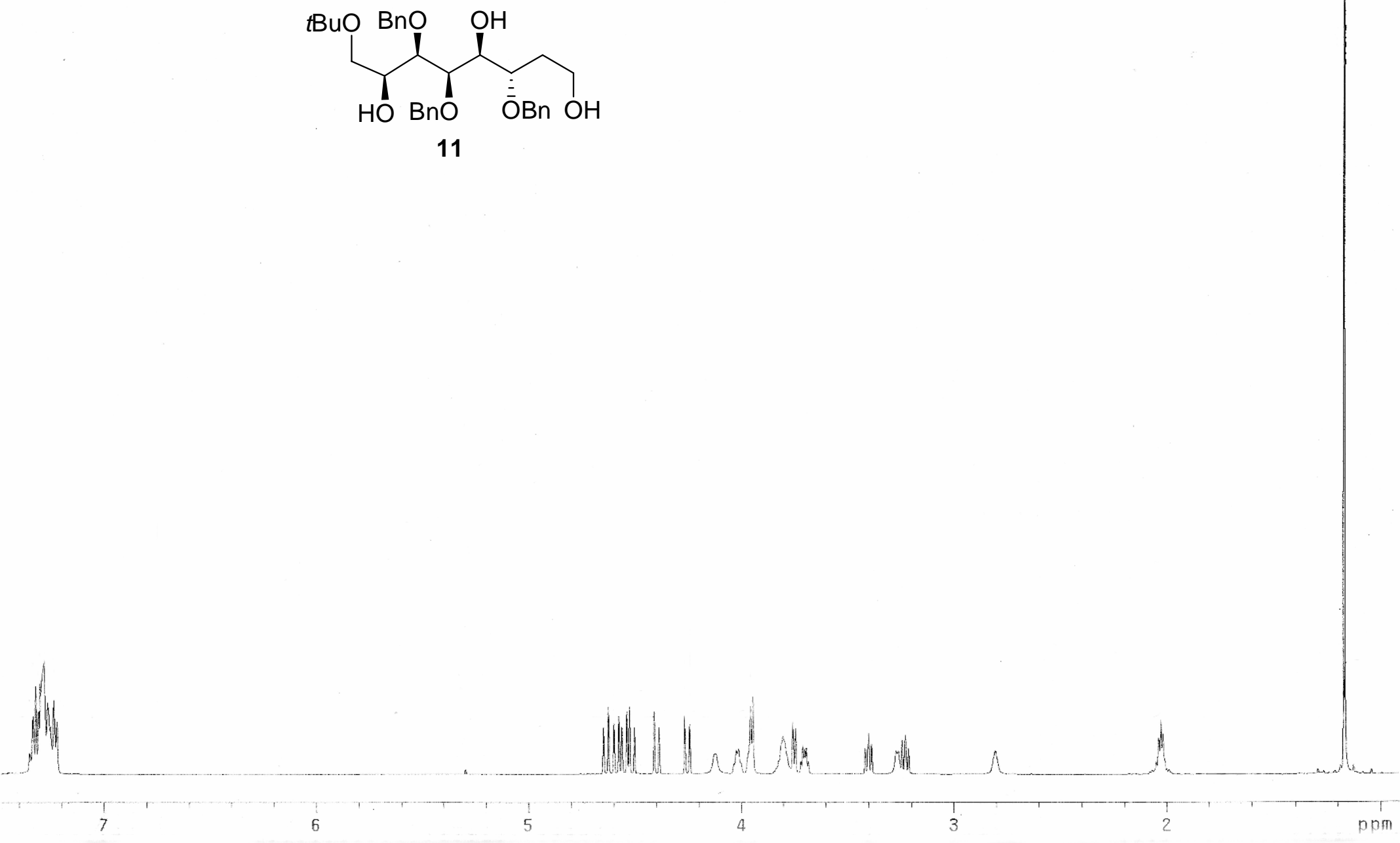

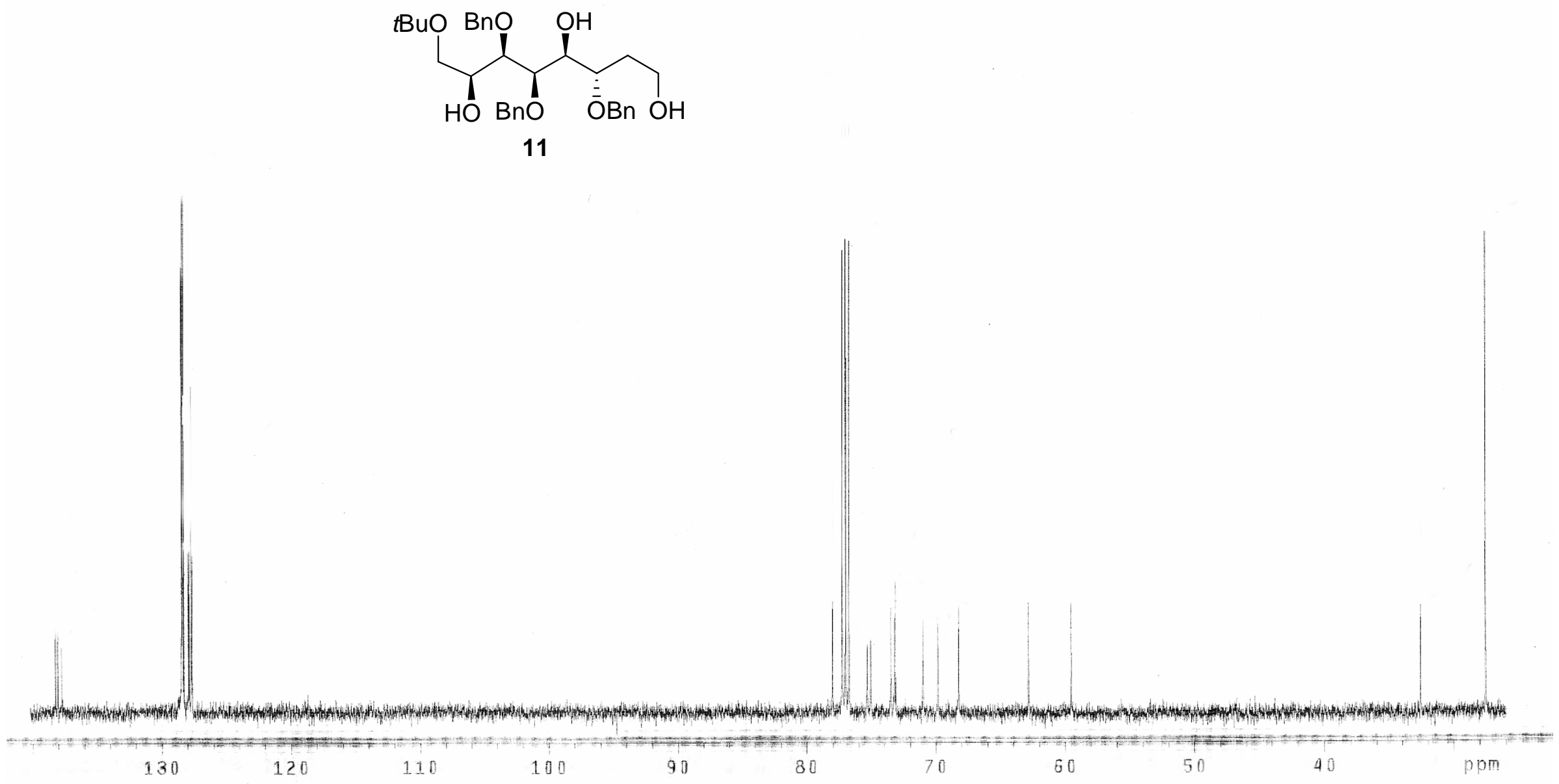


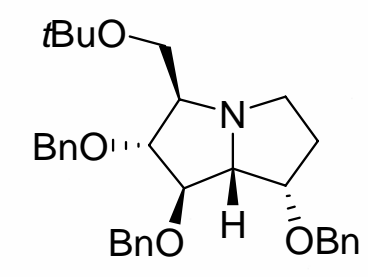

13

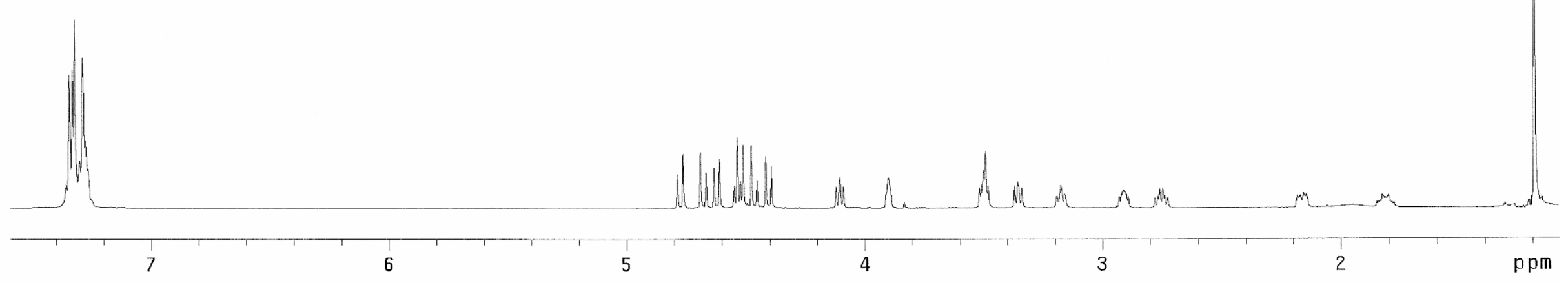




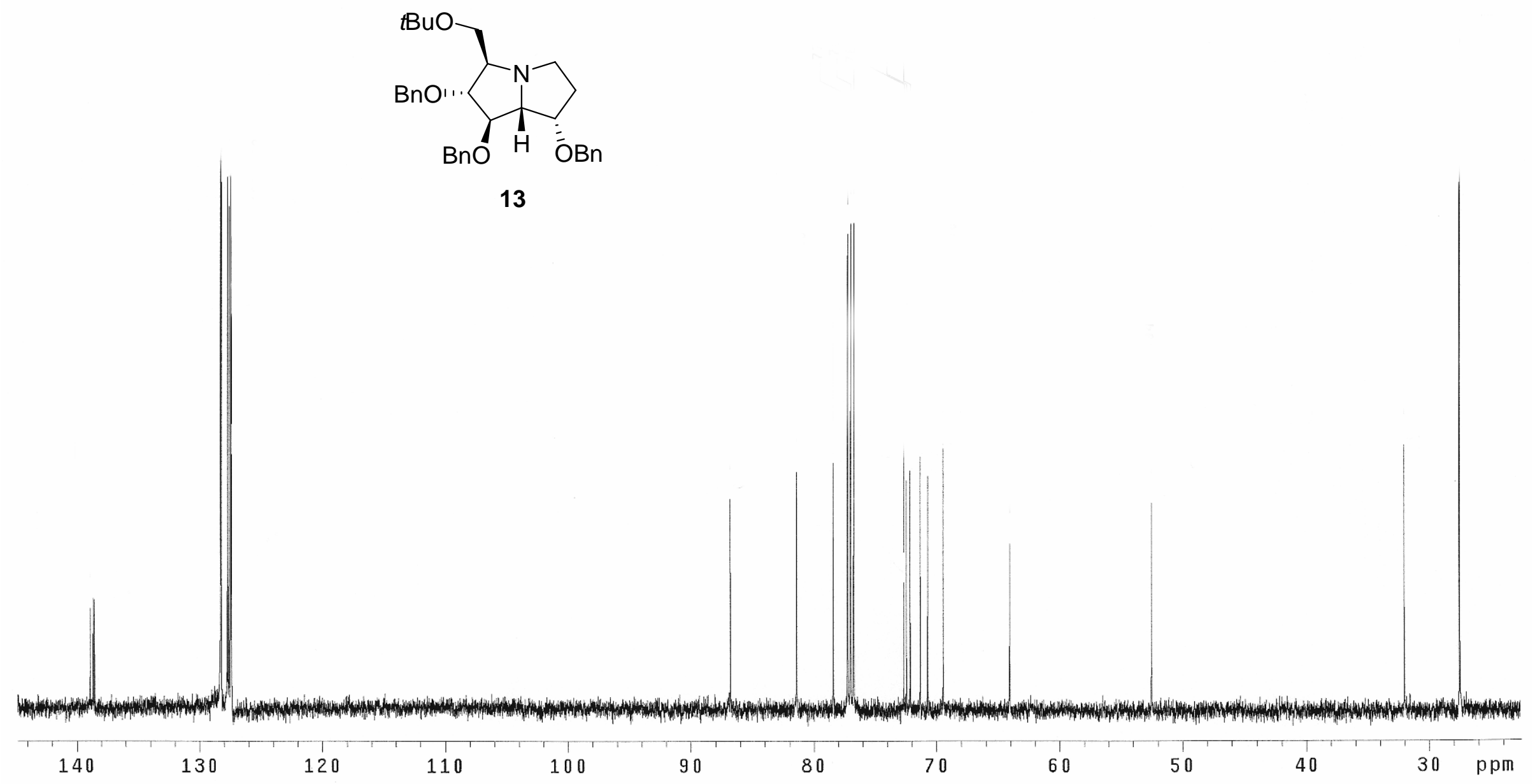




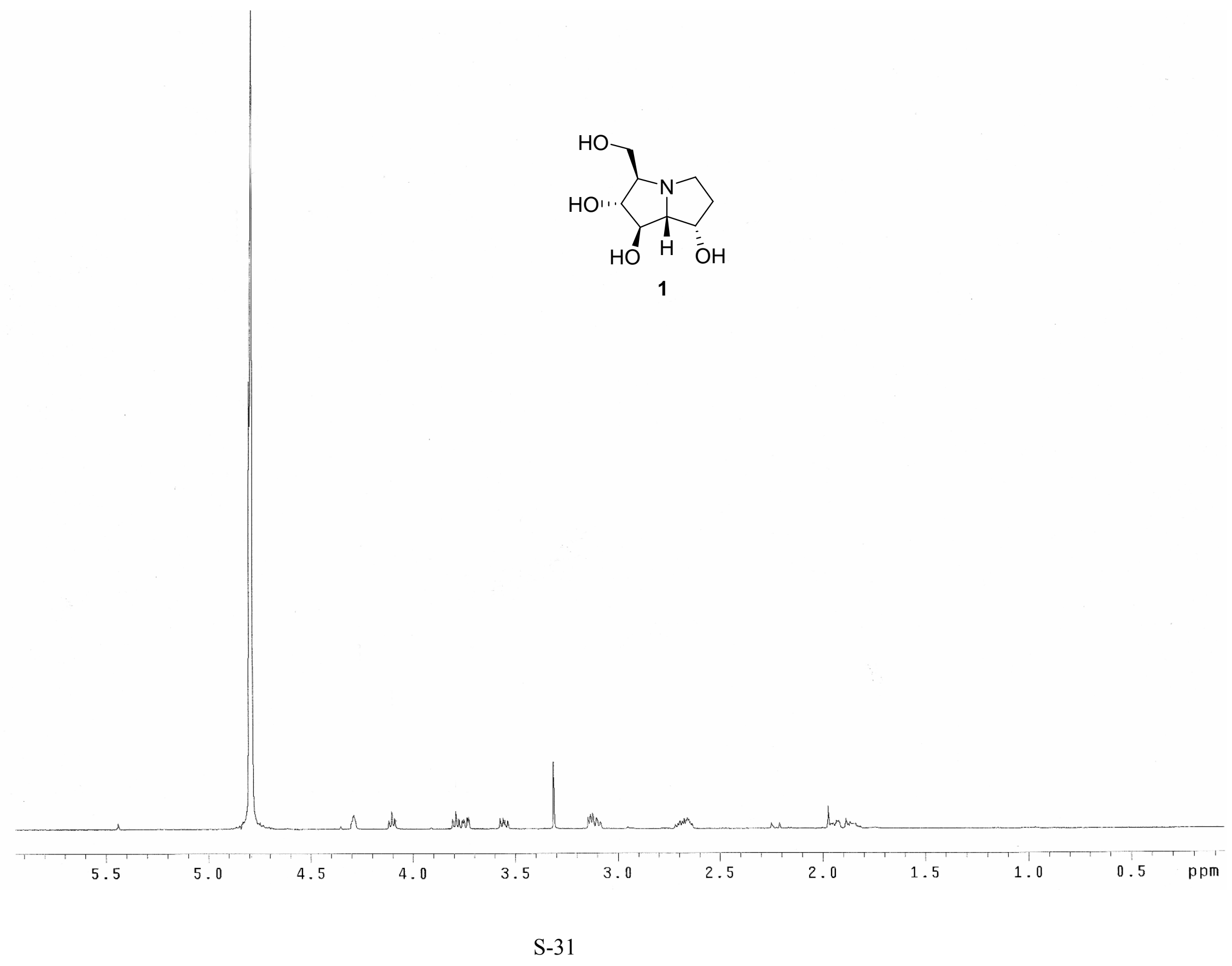




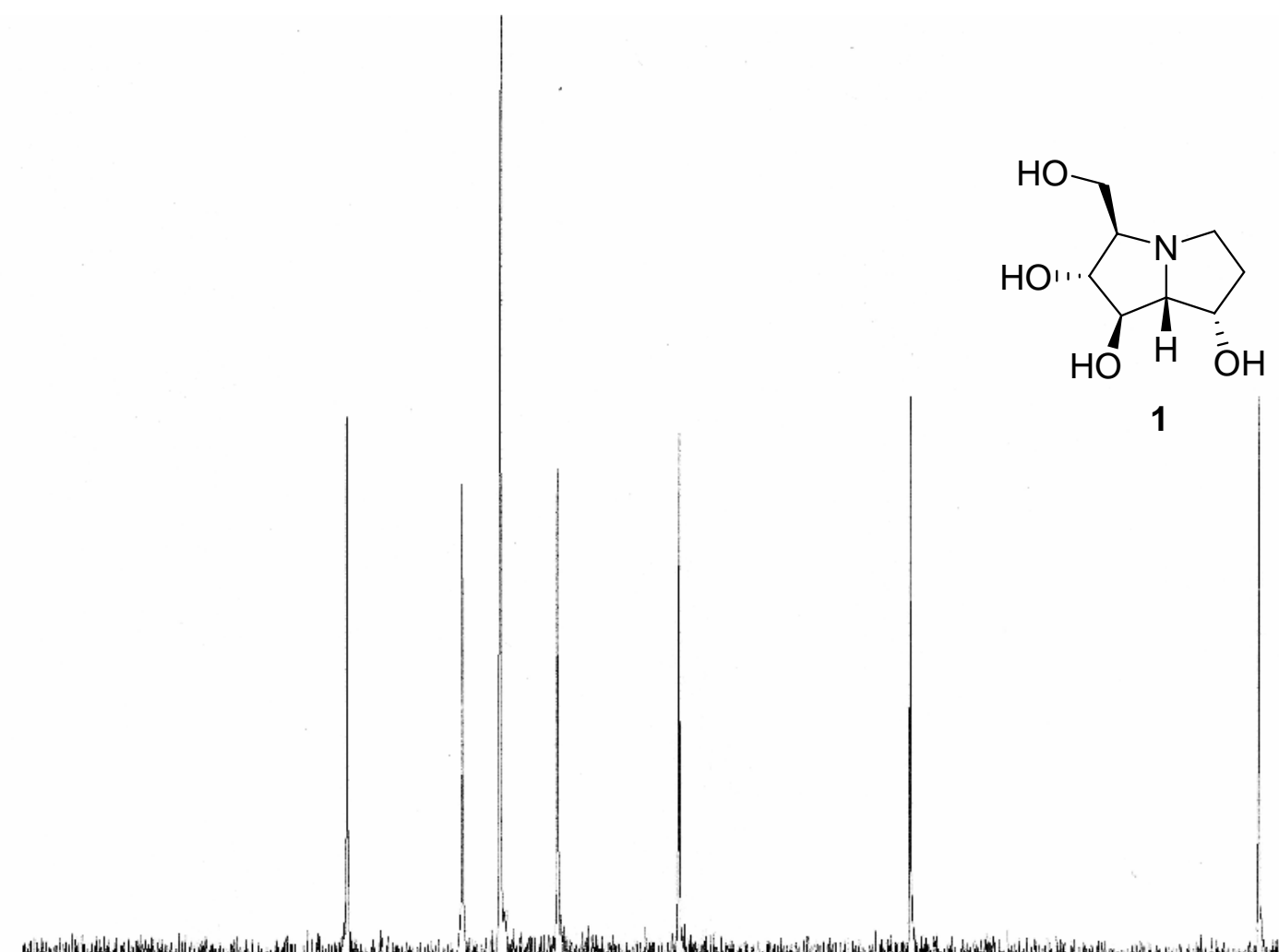

W 